\title{
A Nondominated Sorting Stochastic Fractal Search Algorithm for Multiobjective Distribution Network Reconfiguration with Distributed Generations
}

\author{
Tung Tran The, ${ }^{1,2}$ Bao-Huy Truong $\mathbb{D}^{3},{ }^{3,4}$ Khanh Dang Tuan $\mathbb{D}^{1},{ }^{1,2}$ and Dieu Vo Ngoc $\mathbb{D}^{1,2}$ \\ ${ }^{1}$ Department of Power Systems, Ho Chi Minh City University of Technology (HCMUT), 268 Ly Thuong Kiet Street, District 10, \\ Ho Chi Minh City, Vietnam \\ ${ }^{2}$ Vietnam National University Ho Chi Minh, Linh Trung Ward, Thu Duc District, Ho Chi Minh City, Vietnam \\ ${ }^{3}$ Institute of Engineering and Technology, Thu Dau Mot University, Thu Dau Mot, Binh Duong Province, Vietnam \\ ${ }^{4}$ PEC Technology, 170-170 Bis Bui Thi Xuan Street, Pham Ngu Lao Ward, District 1, Ho Chi Minh City, Vietnam \\ Correspondence should be addressed to Dieu Vo Ngoc; vndieu@hcmut.edu.vn
}

Received 5 November 2020; Revised 4 January 2021; Accepted 22 January 2021; Published 12 February 2021

Academic Editor: Chien Ming Chen

Copyright (C) 2021 Tung Tran The et al. This is an open access article distributed under the Creative Commons Attribution License, which permits unrestricted use, distribution, and reproduction in any medium, provided the original work is properly cited.

\begin{abstract}
This paper aims to propose a new multiobjective algorithm for multiobjective distributed network reconfiguration (DNR) with the placements of distributed generation (DG) in radial distribution networks (RDNs). The new proposed algorithm, called the nondominated sorting stochastic fractal search (NSSFS), is a new multiobjective version of the original SFS algorithm. NSSFS incorporated fast nondominated sorting strategies, crowding distance computation, and selection mechanism into SFS to find and maintain the best nondominated solutions. The proposed NSSFS algorithm was tested with eight multiobjective benchmark test functions to validate its performance. The NSSFS was then implemented to define the optimal network configuration, positions, and sizes of DG units in the RDNs, where real power loss, voltage profile, and voltage stability index were optimized simultaneously. The implementation of multiobjective DNR-DG (MODNR-DG) significantly enhanced the performance of the system. Based on the comparison outcomes, the NSSFS algorithm obtained better solution quality than other multiobjective techniques, proving the effectiveness of NSSFS in dealing with the MODNR-DG problem.
\end{abstract}

\section{Introduction}

The radial distribution networks (RDNs) play a significant role in power systems to transmit electricity to customers. Optimal operation and planning of RDNs pose new challenges for system planners and operators [1]. In order to operate the system optimally, critical factors are considered, such as reducing power loss and improving power quality. Distribution network reconfiguration (DNR) is an effective technique to accomplish these issues [2]. DNR is performed to acquire the optimal configuration to operate the system better while fulfilling the distribution system's operational constraints [3].

The implementation of DNR not only affects power loss but also affects many aspects of the RDNs. Therefore, DNR considering multiobjective function has drawn considerable attention in the literature. In Ref. [4], a multiobjective modified shuffled frog-leaping method was developed for the DNR with multiple objectives, including real power loss, bus voltage deviation, and the number of switching operations. Andervazh et al. [5] used a discrete Particle Swarm Optimization Algorithm with graph theory to optimize three objective functions similar to those in Ref. [4]. In Ref. [6], the authors proposed an efficient hybrid big bang-big crunch technique for the DNR problem of the unbalanced and balanced DNR. A nondominated sorting evolutionary programming method was developed by Hsu and Tsai [7] for the multiobjective DNR problem. The DNR considering reactive power compensators was performed using a Fuzzy Shuffled Frog-Leaping Algorithm in Ref. [8]. In Ref. [9], the 
authors applied the Enhanced Gravitational Search Algorithm for DNR considering to optimize the operation cost, power loss, and enhance the reliability of the network. In Ref. [10], Fireworks Algorithm was applied for voltage profile improvement and power loss minimization. The normalized objectives were formulated as the primary objective, which could not acquire the best solution.

Barbosa et al. [11] implemented the Interval Multiobjective Evolutionary Algorithm to optimize switching operations, power loss, bus voltage deviation, and current index. A Genetic Algorithm was suggested to improve reliability issues and power quality, the solution found by this method based on the selected weights in Ref. [12]. In Ref. [13], the authors suggested a multiobjective approach called Genetic Algorithm-based solver for DNR, where power loss, load balancing index, and energy not supplied were minimized simultaneously. Gravitational Search Algorithm was proposed by Shuaib et al. [14] for DNR with two objectives of voltage deviation and power loss. Shareef et al. [15] developed a Quantum Firefly Algorithm for the DNR problem. Sudha Rani et al. [16] applied Multiobjective Invasive Weed Optimization for DNR problems considering objectives of power loss, load balancing index, number of switching operations, and bus voltage deviation. In Ref. [17], a FuzzyFirefly Algorithm was applied for DNR in unbalanced distribution networks, where the bus voltage deviation, load balancing index, and power loss were optimized simultaneously. In Ref. [18], the Runner-Root Algorithm was applied for the DNR problem. The final compromised solution was selected using the max-min method.

Due to the deregulation of electricity, exhaustion of fossil fuel, and environmental issues, distributed generation (DG) is widely installed in RDNs in recent years. The integration of DG units has significant impacts on the operation of systems. Therefore, numerous studies have investigated the DNR combined with optimal DG placement (ODGP) to enhance systems' quality. Fireworks Algorithm [19] and Harmony Search Algorithm [20] were suggested for the DNR integrated with DG. The voltage profile and real power loss were selected as two objectives for optimization. In Ref. [21], the authors suggested an Adaptive Cuckoo Search Algorithm to solve the DNR considering the ODGP. The graph theory was implemented to check the radial network constraint and reduce infeasible configurations of the network. Adaptive Shuffled Frogs-leaping Algorithm [22] was implemented to consider DNR and ODGP simultaneously. Gong et al. [23] developed a method called the electromagnetismlike mechanism (ELM) to deal with the DNR and ODGP simultaneously. Their objective functions were to minimize voltage stability and power loss. Murty et al. [24] suggested the hybrid method of the General Algebraic Modelling System and Gravitational Search Algorithm for DNR and ODGP problem. The objectives of this problem were to maximize annual cost savings and minimize power loss. The uncertainties of renewablebased DGs and load were also considered in this study. Tolabi et al. [25] employed a hybrid Fuzzy-Bees method for DNR considering ODGP to decrease loss and improve the balance of the feeder load balancing and voltage profile. Muthukumar [26] developed a hybrid method of Particle Artificial Bee Colony Algorithm and Harmony Search Algorithm for DNR considering the optimal positions of shunt capacitors and DG units for voltage profile enhancement, reduction of power loss, and line loading. In Ref. [27], Nondominated Sorting Genetic Algorithm II was used to define positions and settings of DG units in 33-bus RDNs. The results showed that DG improved system performance in terms of real power loss, load voltage deviation, and annualized investment cost. In Ref. [28], a fuzzy multiobjective method was used to handle DNR. After that, a heuristic technique was applied for DNR, which creates a solution based on the original configuration. In Ref. [29], the point estimate method was proposed for multiobjective DNR problems with fuel cells and distributed wind power plants. In Ref. [30], butterfly optimization method was proposed to optimize the power loss, maximum load ability, and loading margin factor.

A review of the literature undertaken found that most of the studies [4-18] only concentrates on the DNR problem. A few studies [19-30] have discussed the DNR considering DG with multiobjective functions, which used traditional methods to convert all objective functions into a single objective function, without considering multiobjective algorithms. However, traditional methods are no longer suitable for dealing with problems with multiobjective functions. The decision-makers need to define all possible optimal solutions for all objective functions concurrently, known as trade-offs. To our best knowledge, very few studies investigated the Pareto optimality concept to define the trade-off solutions for DNR-DG with multiobjective functions. Hence, this motivates us to propose a new method to define trade-off solutions for a combination of DNR and ODGP problem with multiobjective functions using the Pareto dominance concept.

This study aims to develop a new multiobjective method called nondominated sorting stochastic fractal search (NSSFS) for multiobjective DNR with the integration of DG (MODNR-DG) problem. This algorithm integrated three strategies, including fast nondominated sorting strategies, crowding distance computation, and selection mechanism into the original SFS to develop its multiobjective version. The MODNR-DG problem was formulated with three objectives: real power loss, voltage profile, and voltage stability index. The NSSFS was implemented to define the optimal configuration, optimal locations, and sizes of DG units with several cases of objectives in the RDNs.

The contributions of this paper are outlined as follows: 
(1 )A new multiobjective algorithm, namely NSSFS, was developed to deal with the MODNR-DG problem, where real power loss, voltage profile, and voltage stability index were simultaneously optimized.

(2) The proposed NSSFS algorithm was validated on the eight multiobjective benchmark test problems. The obtained results from NSSFS were compared to those from other multiobjective algorithms based on performance metrics. Comparative results showed that Pareto optimal fronts obtained by NSSFS provided better convergence and distribution than other techniques.

(3) The NSSFS was implemented on the 33-bus and 69bus RDNs. Comparison results of NSSFS and other algorithms showed that NSSFS obtained better performance than other multiobjective methods for solution quality.

(4) The quality of RDNs (real power loss, voltage deviation, and voltage stability) was pointedly enhanced after multiobjective optimization.

This paper is structured as follows: Section 2 presents the problem formulation of the MODNR-DG problem. The proposed NSSFS is introduced in Section 3. Afterward, the implementation of NSSFS to the MODNR-DG problem is presented in Section 4 . Section 5 provides the simulation results. Lastly, Section 6 concludes this study.

\section{Problem Formulation}

The MODNR-DG problem aims to optimize real power loss, voltage deviation, voltage stability index simultaneously through the optimal setting of opened switches, positions, and sizes of DG units while fulfilling operational constraints in an RDN. Therefore, the MODNR-DG problem is generally formulated as follows:

$$
\begin{array}{ll}
\text { minimize: } & F(x)=\left[F_{1}(x), F_{2}(x), \ldots, F_{M}(x)\right], \\
\text { subject to: } & g(x) \leq 0, \\
& h(x)=0,
\end{array}
$$

where $F(x)$ is the objective vector of $M$ objective functions; $x$ the vector of design variables; $g(x)$ and $h(x)$ are the set of inequality and equality constraints, respectively.

2.1. Objective Functions. The MODNR-DG problem considers three objective functions as follows.

2.1.1. Real Power Loss Minimization $\left(P_{L}\right)$. The objective to optimize the real power loss of the RDN is defined as follows:

$$
F_{1}=\operatorname{Min}\left(P_{L}\right)=\operatorname{Min}\left(\sum_{k=1}^{N_{L}} R_{k} I_{k}^{2}\right),
$$

where $R_{k}$ is the resistance of the $k^{\text {th }}$ branch, $I_{k}$ is the current passing through that branch, and $N_{L}$ is the number of branches in an RDN.
2.1.2. Voltage Deviation Minimization (VD). The objective to optimize total voltage deviation is given as the following equation:

$$
F_{2}=\operatorname{Min}(\mathrm{VD})=\operatorname{Min}\left[\sum_{i=1}^{N_{B}}\left(V_{i}-V_{r e f}\right)^{2}\right] \text {, }
$$

where $V_{i}$ is the voltage magnitude at the $i^{\text {th }}$ bus, $V_{r e f}$ is the reference voltage (1.0 p.u.), and $N_{B}$ is the number of buses in an RDN.

2.1.3. Voltage Stability Index Maximization (VSI). According to Figure 1, the power of $P_{i+1}$ and $Q_{i+1}$ is defined as follows [31]:

$$
P_{i+1}-j Q_{i+1}=I_{k} \times V_{i+1},
$$

where

$$
I_{k}=\frac{V_{i}-V_{i+1}}{R_{k}+j X_{k}} .
$$

The VSI at $(i+1)^{\text {th }}$ bus can be express as follows [31]:

$$
\mathrm{VSI}_{i+1}=\left|V_{i}\right|^{4}-4\left(P_{i+1} X_{k}-Q_{i+1} R_{k}\right)-4\left(P_{i+1} R_{k}+Q_{i+1} X_{k}\right)\left|V_{i}\right|^{2},
$$

where $X_{k}$ is the resistance of the $k^{\text {th }}$ branch.

The objective to maximize VSI is described as follows:

$$
F_{3}=\operatorname{Max}\left(\operatorname{VSI}_{i+1}\right)=\operatorname{Min}\left(\frac{1}{\operatorname{VSI}_{i+1}}\right) .
$$

2.2. Design Variables. The set of design variables are defined as follows:

$\mathrm{SW}_{i}$ : opened switches in $\mathrm{RDN}$

$L_{\mathrm{DG}, i}$ : location of DG units

$P_{\mathrm{DG}, i}$ : capacity of DG units

Therefore, the vector of design variables $(x)$ is stated as follows:

$$
x=\left[\mathrm{SW}_{1}, \ldots, \mathrm{SW}_{N_{\mathrm{SW}}}, L_{\mathrm{DG}, 1}, \ldots, L_{\mathrm{DG}, N_{\mathrm{DG}}}, P_{\mathrm{DG}, 1}, \ldots, P_{\mathrm{DG}, N_{\mathrm{DG}}}\right],
$$

where $N_{\mathrm{SW}}$ is the number of opened switches, and $N_{\mathrm{DG}}$ is the number of DG units to be installed.

2.3. System Constraints. The operational constraints for the MODNR-DG problem are given as follows.

2.3.1. Equality Constraints. The real and reactive powers of an RDN must be conserved as follows: 


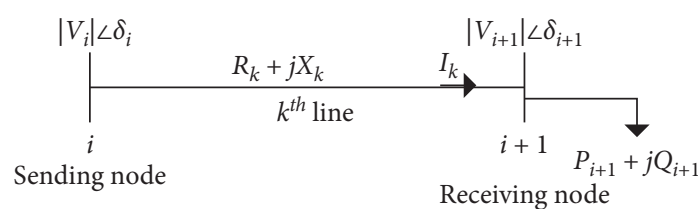

Figure 1: Schematic view of a single line in the RDN.

$$
\begin{aligned}
P_{\mathrm{SS}}+\sum_{i=1}^{N_{\mathrm{DG}}} P_{\mathrm{DG}, i} & =\sum_{j=1}^{N_{B}} P_{D, j}+\sum_{k=1}^{N_{L}} P_{L, k}, \\
\mathrm{Q}_{\mathrm{SS}}+\sum_{i=1}^{N_{\mathrm{DG}}} \mathrm{Q}_{\mathrm{DG}, i} & =\sum_{j=1}^{N_{B}} Q_{D, j}+\sum_{k=1}^{N_{L}} \mathrm{Q}_{L, k},
\end{aligned}
$$

where $P_{S S}$ and $Q_{S S}$ are the real and reactive powers supplied from the slack bus, respectively; $P_{\mathrm{DG}, i}$ and $Q_{\mathrm{DG}, i}$ are the real and reactive power outputs of the $i^{\text {th }}$ DG unit, respectively; $P_{D, j}$ and $Q_{D, j}$ are the real and reactive power of load demands at the ${ }^{j \text { th }}$ bus, respectively; $P_{L, k}$ and $Q_{L, k}$ are the real and reactive power losses in the $k^{\text {th }}$ branch, respectively.

\subsubsection{Inequality Constraints}

(i) Voltage constraint: the bus voltage amplitude is restricted by the boundaries as follows:

$$
V_{\min , i} \leq V_{i} \leq V_{\max , i} ; \quad i=1, \ldots, N_{B}
$$

where $V_{\min , i}$ and $V_{\max , i}$ are the voltage limits at the $i^{\text {th }}$ bus.

(ii) Thermal limit: the current flows through a branch must not violate the following restriction:

$$
\left|I_{k}\right| \leq\left|I_{\max , k}\right| ; \quad k=1, \ldots, N_{L}
$$

where $I_{\max , k}$ is the maximum current allowed to flow through the $k^{\text {th }}$ branch.

(iii) DG generation constraint: the minimum and maximum limits restrict the power output of DG units as follows:

$P_{\mathrm{DG} \min , i} \leq P_{\mathrm{DG}, i} \leq P_{\mathrm{DG} \max , i} ; \quad i=1, \ldots, N_{\mathrm{DG}}$,

where $\mathrm{P}_{\mathrm{DGmin}, i}$ and $P_{\mathrm{DGmax}, i}$ are the ith DG unit's limited sizes.

(iv) DG penetration constraint: the level penetration of DG units has restrictions as follows:

$$
\sum_{i=1}^{N_{\mathrm{DG}}} P_{\mathrm{DG}, i} \leq \sum_{j=1}^{N_{B}} P_{D, j}
$$

(v) Radial configuration constraint: the distribution network must ensure the radial configuration and serve all loads after DNR as follows:

$$
\operatorname{det}(A)=1 \text { or }-1
$$

where $\mathrm{A}$ is a matrix representing the connections of branches and buses in the RDN.

\section{Nondominated Sorting Stochastic Fractal Search Algorithm}

The proposed NSSFS algorithm was developed based on strategies of NSGA-II to dealing with multiobjective problems effectively. Strategies of NSGA-II, namely, fast nondominated sorting and crowding distance, and selection procedures have been prevalent and applied in many recent works such as [32-37]. The original SFS, new integration methods, and the new NSSFS are now presented.

3.1. Stochastic Fractal Search Algorithm. The SFS is a robust metaheuristic algorithm, which was developed by Salimi [38]. SFS applies an idea called the fractal to simulate the natural phenomenon of growth. The SFS method contains two main phases: diffusion and updating processes.

3.1.1. Diffusion Process. Each particle diffuses around its current location to promote exploitation ability. The strategy here is to intensify the probability of obtaining the global minimum and avoid being stuck in the local minima. Generally, new particles are generated via a series of Gaussian walks participating according to

$$
\begin{cases}X_{i, \text { new }}=\operatorname{Gaussian}\left(\mu_{X_{\text {best }}} \sigma\right)+\left(\varepsilon \times X_{\text {best }}-\varepsilon^{\prime} \times X_{i}\right), & \text { if rand } \leq W, \\ X_{i, \text { new }}=\operatorname{Gaussian}\left(\mu_{X}, \sigma\right), & \text { otherwise, }\end{cases}
$$

where $X_{i \text {, new }}$ is the new location of $i^{\text {th }}$ particle; $X_{i}$ and $X_{\text {best }}$ indicate the location of the $i^{\text {th }}$ particle and the best particle of the population, respectively; $\varepsilon$ and $\varepsilon^{\prime}$ are stochastic numbers between a range $[0,1] ; W$ is the Gaussian walk parameter; $\mu_{X}$ is equal to $X_{i}, \mu_{\text {best }}$ is equal to $X_{\text {best }}$, and the parameter $\sigma$ is computed as follows:

$$
\sigma=\left|\frac{\log (G)}{G} \times\left(X_{i}-X_{\text {best }}\right)\right|,
$$

where $G$ is the number of iterations. As $\log (G) / G$ decreases in value through iterations, the magnitude of Gaussian jumps also decreases. This enhances the ability of the search to be more localized and closer to the optimum solution.

3.1.2. Updating Process. In this process, the first statistical method is implemented to each particle, while the second statistical procedure is then performed on all particles. The purpose of the two procedures is to increase the exploration of the SFS algorithm.

(1) The First Statistical Procedure. All particles of the population are sorted depending on the objective function 
values. A probability value is then given for each particle $X_{i}$ as follows:

$$
\mathrm{Pa}_{i}=\frac{\operatorname{rank}\left(X_{i}\right)}{N_{p}}
$$

where $N_{p}$ is the population size, and $\operatorname{rank}\left(X_{i}\right)$ denotes the rank of particle $X_{i}$ in the population.

An aspect worth mentioning is that a better particle has a higher probability. Therefore, the particles having the worst objective function values are more likely to be changed. For each particle $X_{i}$ in population, if the condition $P \mathrm{a}_{i}<\varepsilon$ is fulfilled, the $j$ th component of $X_{i}$ is modified via equation (18); otherwise, it remains unchanged.

$$
X_{i}^{\prime}(j)=X_{r}(j)-\varepsilon \times\left(X_{t}(j)-X_{i}(j)\right),
$$

where $X_{i}^{\prime}$ is a newly modified particle from particles; $X_{i}, X_{r}$, and $X_{t}$ are randomly selected particles in the population, and $\varepsilon$ is the stochastic variable.

The new particle $X_{i}^{\prime}$ will replace the position of $X_{i}$ if its objective value is better than $X_{i}$.

(2) The Second Statistical Procedure. Similarly, particles yielded by the first statistical process are sorted according to equation (17). If the condition $P a_{i}<\varepsilon^{\prime}$ is fulfilled, the current location of $X_{i}^{\prime}$ is modified by equation (19); otherwise, it remains unchanged.

$$
\begin{cases}X_{i}^{\prime \prime}=X_{i}^{\prime}-\varepsilon^{\prime} \times\left(X_{t}^{\prime}-X_{\text {best }}\right), & \text { if } \varepsilon^{\prime} \leq 0.5, \\ X^{\prime \prime}=X_{i}^{\prime}+\varepsilon^{\prime} \times\left(X_{t}^{\prime}-X_{r}^{\prime}\right) & \text { if } \varepsilon^{\prime}>0.5,\end{cases}
$$

where $X_{i}^{\prime \prime}$ is a newly modified particle from particle; $X_{i}^{\prime}, X_{r}^{\prime}$, and $X_{t}^{\prime}$ are randomly selected particles yielded from the first procedure; $\varepsilon^{\prime}$ and $\varepsilon^{\prime \prime}$ are the stochastic variables.

The new particle $X_{i}^{\prime \prime}$ will replace the position of $X_{i}^{\prime}$ if its objective value is better than $X_{i}^{\prime}$.

3.2. Fast Nondominated Sorting Technique. This technique is used to determine the nondominated ranks of a population. To identify the nondominated ranks, two quantitative measures (domination count $n_{p}$ and a set $S_{p}$ ) are calculated for each solution in the population, where $n_{p}$ is the number of solutions that dominate the solution $p$, and $S_{p}$ is a set that contains all solutions that are dominated by solution $p$. The solutions that are not dominated by any solutions $\left(n_{p}=0\right)$ are placed in the first nondominated rank. After that, domination count $n_{q}$ is reduced by one for each solution $q$ belonging to the set $S_{p}$. If $n_{q}=0$, the corresponding solution $q$ is placed in the second nondominated rank. Similarly, the process proceeds until all solutions are placed in a nondominated rank.

3.3. Crowding Distance Computation. In order to preserve the diversity among solutions in a particular nondominated rank, the crowding distance computation is applied. All solutions are ranked depending on the value of each objective. Infinite values are assigned to crowding distances of boundary solutions. The crowding distance of solution $i$, which is surrounded by solutions $(i-1)$ and $(i+1)$ is defined as follows:

$$
d_{i}=\sum_{j=1}^{k} \frac{f_{i+1}^{j}-f_{i-1}^{j}}{f_{\max }^{j}-f_{\min }^{j}},
$$

where $k$ is the number of objectives; $f_{\max }^{j}$ and $f_{\min }^{j}$ are the maximum and minimum values of the $j^{\text {th }}$ objective for the boundary solutions; $f_{i-1}^{j}$ and $f_{i+1}^{j}$ are the values of the $j^{\text {th }}$ objective for the solutions $(i-1)$ and $(i+1)$, respectively.

Each solution $i$ has two qualitative measures: nondomination rank $r_{i}$ and crowding distance $d_{i}$. A crowded comparison operator (denoted by $\prec_{n}$ ) is applied to compared two solutions $i$ and $j$ as follows:

$$
i \prec_{n} j, \quad \text { if }\left(r_{i}<r_{j}\right) \text { or }\left(\left(r_{i}=r_{j}\right) \text { and }\left(d_{i}>d_{j}\right)\right) \text {. }
$$

Based on equation (21), it can be indicated that between two solutions $i$ and $j$, the solution with the lower rank will be preferred. If both solutions have the same rank, the solution with the higher crowding distance value will be preferred.

3.4. Selection Procedure. The selection procedure is an essential task of MOO to generate a good Pareto optimal front. In the SFS algorithm, after each process, the new particles will replace the current particles if their objective function values are better than those of the current particles. The current particles are then discarded. However, in the NSSFS algorithm, after new particles are created in each process, they are compared to current particles via a crowded comparison operator. If the new particle dominates the current one, it will replace the current particle immediately, and the current particle will be added to the extra population. Otherwise, the new particle will be added to the extra population. In this way, all solutions are retained to avoid losing potential candidate solutions. After each iteration, the two populations (current and extra) are merged, after which the merged population size is larger than the population size $\left(N_{p}\right)$. However, the population size must be maintained at $N_{p}$ during the optimization process. Therefore, a selection mechanism of $N_{p}$ best particle is performed, which is shown in Figure 2.

Firstly, a fast nondominated sorting method is used to sort the merged population into different nondominated fronts $\left(F_{1}, F_{2}, \ldots, F_{n}\right)$. The best solutions in the first front $F_{1}$ are first chosen for inclusion in the new population. If the number of solutions from $F_{1}$ is smaller than $N_{p}$, the remaining solutions of the new population will be designated from subsequent fronts $\left(F_{2}, \ldots, F_{n}\right)$ in the order of their nondomination rank. This procedure continues until the population is filled with $N_{p}$ solutions. Assume that the front $F_{1}$ is the last front, solutions in this front are selected based on the crowding distance value to select precisely $N_{p}$ solutions for the new population.

3.5. NSSFS Algorithm. The NSSFS algorithm begins the multiobjective optimization process by creating an initial population of $N_{p}$ particles. Objective function values are 
then defined for each particle in the initial population. Based on fast nondominated sorting and crowding distance techniques, the best particle is selected for the next two processes (diffusion and updating processes). After each iteration, the extra population is obtained, and the current and extra populations are merged. The selection procedure is then performed to select $N_{p}$ best particles for the next generation. The optimization process of NSSFS repeats until the stopping criteria are fulfilled.

The pseudocode of the NSSFS algorithm (Algorithm 1) is given in Figure 3.

\section{Implementation of NSSFS to MODNR- DG Problem}

4.1. Initialization of Population. For the implementation of the NSSFS algorithm to the MODNR-DG problem, each particle of the initial population denotes a vector of design variables, including positions of opened switches, positions, and sizes of DG units as follows:

$$
X_{i}=\left[\mathrm{SW}_{1}, \ldots, \mathrm{SW}_{N_{\mathrm{SW}}}, L_{\mathrm{DG}, 1}, \ldots, L_{\mathrm{DG}, N_{\mathrm{DG}}}, P_{\mathrm{DG}, 1}, \ldots, P_{\mathrm{DG}, N_{\mathrm{DG}}}\right], \quad \text { for } i=1, \ldots, N_{p} \text {. }
$$

Each particle is randomly created within the boundaries. Therefore, the design variables for locations of opened switches $\left(\mathrm{SW}_{i}\right)$, locations $\left(L_{\mathrm{DG}, i}\right)$, and sizes $\left(P_{\mathrm{DG}, i}\right)$ of $\mathrm{DG}$ units are created according to the equations as follows:

$$
\begin{aligned}
& \mathrm{SW}_{i}=\operatorname{round}\left[\mathrm{SW}_{\mathrm{min}, i}+\operatorname{rand}(0,1) \times\left(\mathrm{SW}_{\mathrm{max}, i}-\mathrm{SW}_{\mathrm{min}, i}\right)\right], \\
& L_{\mathrm{DG}, i}=\operatorname{round}\left[L_{\mathrm{DG} \min , i}+\operatorname{rand}(0,1) \times\left(L_{\mathrm{DG} \max , i}-L_{\mathrm{DG} \min , i}\right)\right], \\
& P_{\mathrm{DG}, i}=P_{\mathrm{DG} \min , i}+\operatorname{rand}(0,1) \times\left(P_{\mathrm{DG} \max , i}-P_{\mathrm{DG} \min , i}\right) .
\end{aligned}
$$

4.2. Objective Function Value. Each objective function value of the MODNR-DG problem is calculated as follows:

$$
\begin{aligned}
F_{m}^{\prime}= & F_{m}+K_{p} \sum_{i=1}^{N_{B}}\left(V_{i}-V_{i}^{\mathrm{lim}}\right)^{2}+K_{q} \sum_{k=1}^{N_{L}}\left(I_{k}-I_{k}^{\lim }\right)^{2} \\
& +K_{v}\left(\mathrm{PE}_{\mathrm{DG}}-\mathrm{PE}_{\mathrm{DG}}^{\mathrm{lim}}\right)^{2},
\end{aligned}
$$

where $F_{m}$ is the $m^{\text {th }}$ objective function of the MODNR-DG problem. $K_{p}, K_{q}$, and $K_{v}$ represent penalty coefficients for voltage, thermal, and DG penetration constraints, respectively; $x^{\lim }$ is the limit value of the dependent variable $x$ (bus voltages, currents, and DG penetration), which is given as follows:

$$
x^{\lim }= \begin{cases}x_{\max }, & \text { if } x>x_{\max } \\ x_{\min }, & \text { if } x<x_{\min } \\ x, & \text { otherwise }\end{cases}
$$

where $x$ signifies the $V_{i}, I_{k}$, and $P E_{\mathrm{DG}}$ values; $x^{\text {lim }}$ signifies the limitations of $V_{i}, I_{k}$, and $P E_{\mathrm{DG}}$.

Moreover, the power flow problem is solved by using Matpower 4.1 toolbox to compute the objective function value of each particle $[39,40]$.

4.3. Best Compromise Solution Based on Fuzzy Decision. A fuzzy membership method is used to yield the best compromise solution among final nondominated solutions.
The membership function $\mu_{i j}$ denotes each objective function of the $i$ th solution as follows [41]:

$$
\mu_{i j}= \begin{cases}1, & \text { if } F_{i j} \leq \min \left(F_{j}\right), \\ \frac{\max \left(F_{j}\right)-F_{i j},}{\max \left(F_{j}\right)-\min \left(F_{j}\right)}, & \text { if } \min \left(F_{j}\right) \leq F_{i j} \leq \max \left(F_{j}\right), \\ 0, & \text { if } F_{i j} \geq \max \left(F_{j}\right),\end{cases}
$$

where $\max \left(F_{j}\right)$ and $\min \left(F_{j}\right)$ are maximum and minimum values of the $j^{\text {th }}$ objective function, respectively. The normalized membership function for each solution is given as follows:

$$
\mu_{i}=\frac{\sum_{j=1}^{n_{\mathrm{obj}}} \mu_{i j}}{\sum_{i=1}^{n_{\mathrm{pf}}} \sum_{j=1}^{n_{\mathrm{obj}}} \mu_{i j}},
$$

where $n_{\mathrm{pf}}$ and $n_{\mathrm{obj}}$ are numbers of nondominated solutions and objective functions, respectively. The best compromise solution is the one with the greatest value of $\mu_{i}$.

4.4. Overall Procedure. The implementation of the NSSFS method for the MODNR-DG problem is outlined as follows:

Step 1. Select parameters of NSSFS $\left(N_{p}, N_{D}, G\right.$, and $\left.W\right)$. 


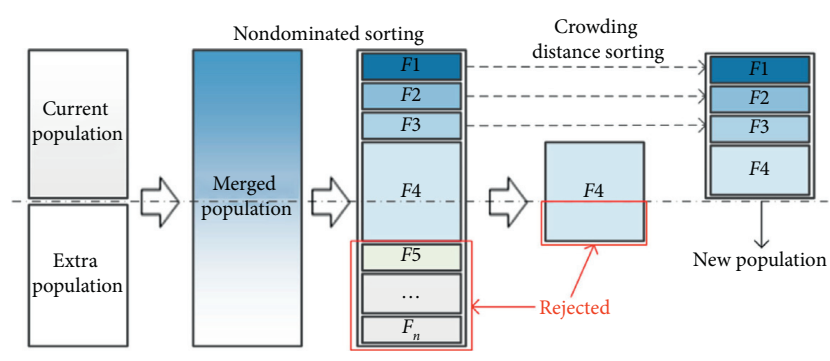

FIgURE 2: Schematic view of the selection procedure.

Step 2. Generate the initial population of $N_{p}$ particles according to equations (23)-(25). Compute objective function values according to equation (26) for all particles of population.

Step 3. Sort population into different nondominated ranks via fast nondominated sorting technique and crowding distance computation. Set $g=0$.

Step 4. Set $g=g+1$.

Step 5. Select the particle which has the best crowding distance value from the first nondominated front as the best particle $\left(X_{\text {best }}\right)$.

Step 6. Perform the diffusion process as follows:

Generate new solutions following equation (15). The best-created solution from the diffusion process for each particle $\left(X_{i}\right)$ is retained as $X_{\text {inew, }}$ and the remaining solutions are added into the extra population.

Update the best particle $\left(X_{\text {best }}\right)$.

Step 7. Perform the updating process as follows:

All solutions are assigned the value of $\mathrm{Pa}_{i}$ according to equation (17).

Generate new solutions following equations (18) and (19).

If the new particle dominates the current particle, it will immediately replace the current population, and the current particle will be added to the extra population. Otherwise, the new particle will be added to the extra population.

Update the best particle $\left(X_{\text {best }}\right)$.

Step 8. Merge the current and extra population. Select best $N_{p}$ solutions from the merged population for the new population via selection mechanism.

Step 9. If $g<G$, return to Step 4. Otherwise, go to Step 10.

Step 10. Define the best compromise solution using the decision-making method.

4.5. Performance Measures. To quantitatively evaluate the performance of a multiobjective method, performance metrics are taken into consideration: (1) generational distance metric; (2) spacing metric; (3) spread metric; (4) hypervolume metric.
4.5.1. Generational Distance Metric. The generational distance (GD) metric measures the convergence of Pareto optimal front generated $\left(\mathrm{PF}_{q}\right)$ by a multiobjective algorithm [42] as follows:

$$
\mathrm{GD}=\frac{\sqrt{\sum_{i=1}^{n_{\mathrm{pf}}} d_{i}^{2}}}{n_{\mathrm{pf}}},
$$

where $n_{\mathrm{pf}}$ is the number of nondominated solutions in $P F_{g}$, and $d_{i}$ is the Euclidean distance between each solution in $P F_{g}$ and the nearest solution in true Pareto front $\left(\mathrm{PF}_{\text {true }}\right)$. A lower value for the GD metric shows better convergence of a multiobjective algorithm.

4.5.2. Spacing Metric. The spacing (SP) metric measures the distribution of solutions in $\mathrm{PF}_{g}$ as follows [43]:

$$
\mathrm{SP}=\sqrt{\frac{1}{n_{\mathrm{pf}}-1} \sum_{i=1}^{n_{\mathrm{pf}}}\left(d_{i}-\bar{d}\right)^{2}},
$$

where $d_{i}=\min _{j}\left(\left|f_{1}^{i}(x)-f_{1}^{j}(x)\right|-\left|f_{2}^{i}(x)-f_{2}^{j}(x)\right|\right), i, j=$ $1,2, \ldots, n_{\mathrm{pf}}$ and $\bar{d}$ is the mean value of all $d_{i}$. A lower value for the SP metric denotes better distribution of a multiobjective algorithm.

4.5.3. Spread Metric. The spread $(\Delta)$ metric measures the extent of spread yielded by solutions in $\mathrm{PF}_{g}$ as follows [44]:

$$
\Delta=\frac{d_{f}+d_{l}+\sum_{i=1}^{n_{\mathrm{pf}}}\left|d_{i}-\bar{d}\right|}{d_{f}+d_{l}+\left(n_{\mathrm{pf}}-1\right) \bar{d}}
$$

where $d_{f}$ and $d_{l}$ are the Euclidean distances between the extreme solutions in $\mathrm{PF}_{\text {true }}$ and $\mathrm{PF}_{q}$, respectively; $d_{i}$ is the Euclidean distance between neighboring solutions in $\mathrm{PF}_{g}$, and $\bar{d}$ is the mean value of all $d_{i}$. A lower value for the $\Delta$ metric shows a better spread of a multiobjective algorithm.

4.5.4. Hypervolume Metric. The hypervolume (HV) metric measures the volume covered by solutions in $\mathrm{PF}_{g}$ in the objective space for a multiobjective problem with two objectives. This metric is given as follows [44]:

$$
\mathrm{HV}=\bigcup_{i=1}^{|\Omega|} v_{i}
$$

A multiobjective algorithm with the highest HV metric obtains a better nondominated set.

\section{Simulation Results}

5.1. Results for Multiobjective Benchmark Problems. In this section, the performance of NSSFS was validated with eight benchmark test functions. These problems consist of wellknown Zitzler-Deb-Thiele's test functions (ZDT1, ZDT2, ZDT3, and ZDT6) [45], Kursawe's test function (KUR) [46], Laumanns's test function (LAU) [47], Murata's test function (MUR) [48], and Poloni's test function (POL) [49]. The NSSFS was executed in MATLAB programming software. 
1: Set control parameters: number of particles $\left(N_{p}\right)$, number of diffusions $\left(N_{D}\right)$, maximum number of iterations number $(G)$, and Gaussian walk parameters $(W)$.

2: $\quad$ Initialize randomly the population $\left(\mathbf{X}=\left[X_{1}, X_{2}, \ldots, X_{N_{p}}\right]^{\mathrm{T}}\right.$;

3: Calculate fitness value for each organism $X_{i}\left(i=1, \ldots, N_{p}\right)$ in the population;

4: Define the best particle $\left(X_{\text {best }}\right)$ which have best crowding distance value from first nondominated front;

5: $\quad$ Set $g=0$;

6: While $(g<G)$

$\%$ Diffusion process \%

7: $\quad$ for $i=1: N_{p}$

8: $\quad$ for $m=1: N_{D}$

9: $\quad$ Create a new particle based on equation (15);

10: $\quad$ The best created solution from diffusion process for each particle $\left(X_{i}\right)$ is retained as

$X_{\text {inew }}$ and the remaining solutions are added into extra population;

11: $\quad$ end

12: $\quad$ Update the best particle $\left(X_{\text {best }}\right)$;

13: $\quad$ End

\% Fist Updating Process \%

14: $\quad$ All points are ranked based on equation (17);

15: $\quad$ for $i=1: N_{p}$

if $\operatorname{rand}(0,1)>P a_{i}$

Update the component based on equation (18);

else

Do nothing;

end

end

If new particle dominates current particle, it will replace immediately in the current population and the current particle will be added to extra population. otherwise, the new particle will be added to extra population;

23: Update the best particle $\left(X_{\text {best }}\right)$;

$\%$ Second Updating Process \%

24: $\quad$ All particles obtained by the first update process are ranked using equation (17);

25: $\quad$ for $i=1: N_{p}$

26: $\quad$ if rand $(0,1)>P a_{i}$

27: $\quad$ Update the component based on equation (19);

28: $\quad$ else

Do nothing;

end

end

If new particle dominates current particle, it will replace immediately in the current population and the current particle will be added to extra population. otherwise, the new particle will be added to extra population;

33: Update the best particle $\left(X_{\text {best }}\right)$;

34: $\quad$ Merge the current and extra population. Select best $N_{p}$ solutions from merged population for new population based on selection mechanism;

35: $\quad g=g+1$;

36: End

Figure 3: Pseudocode of the NSSFS algorithm.

Obtained results were compared with NSGA-II [50], MOPSO [51], and MOMVO [52]. For a fair comparison, the number of function evaluations (NFEs) was 10,000 (stop criteria). Each multiobjective algorithms were carried out thirty times for each benchmark problem. The initial parameters of all algorithms are given in Table 1.
Table 2 presents the statistical results of the GD, SP, and $\Delta$ metrics for NSSFS, NSGA-II, MOPSO, and MOMVO algorithms. As per the results of the GD metric in Table 2, it was evident that NSSFS was able to outperform other algorithms significantly. In fact, NSSFS yielded the best statistical results for the GD metric for all test functions, 
TABLE 1: Initial parameters of NSSFS, NSGA-II, MOPSO, and MOMVO algorithms.

\begin{tabular}{lc}
\hline Algorithms & Parameter settings \\
\hline NSSFS & Population size $\left(N_{p}\right)=100$ \\
& Number of diffusion $\left(N_{D}\right)=5$ \\
Gaussian walk parameter $(W)=0.75$ \\
\hline \multirow{2}{*}{ NSGA-II } & Population size $=100$ \\
& Crossover operator $=20$ \\
& Mutation operator $=20$ \\
MOPSO & Population size $=100$ \\
& Inertia weight $=0.4$ \\
& Adaptive grid $=30$ \\
& Mutation rate $=0.5$ \\
\hline
\end{tabular}

MOMVO Worm hole existence probability $\max =1$

Worm hole existence probability $\min =0.2$

showing a higher convergence of NSSFS compared to other algorithms. Moreover, the NSSFS showed the best performance in obtaining the lowest SP values for all test functions and was placed in the first rank for the SP metric. Regarding $\Delta$ metric, the NSSFS obtained very competitive results in comparison with the other algorithms and occasionally outperformed them for the majority of test functions (six out of eight in Table 2). NSSFS had the advantage of obtaining the best values of $\Delta$ metric for test functions ZDT1, ZDT2, ZDT3, ZDT6, KUR, and POL, while the MOPSO and NSGA-II obtained better values of $\Delta$ metric for test functions LAU and MUR. Therefore, the proposed NSSFS proved superiority over NSGA-II, MOPSO, and MOMVO for all benchmark test functions, especially for the GD metric.

Figures 4 and 5 illustrate $\mathrm{PF}_{\text {true }}$ and $\mathrm{PF}_{g}$ of NSSFS for all test problems. As seen in Figures 4 and 5, Pareto optimal solutions of NSSFS successfully covered the $\mathrm{PF}_{\text {true }}$ with satisfactory distribution and spread.

5.2. Results for MODNR-DG Problem. The NSSFS was carried out on 33-bus and 69-bus RDNs. Moreover, six different case studies were formulated by two or three objective functions defined in equations (2), (3), and (7). The initial parameters of NSSFS $\left(N_{p}, N_{D}\right.$, and $\left.W\right)$ were selected as 100 , 5 , and 0.75 , respectively, for all case studies. The parameter $G$ was set to 200 for Cases 1-3 and 400 for Cases 4-6. The NSSFS method was executed thirty trials independently for each case study to acquire the best solution.

5.2.1. 33-Bus RDN. The proposed NSSFS method was first implemented on the 33-bus RDN. Detailed data can be derived from [53]. For the initial case, the network has a real power loss of $202.68 \mathrm{MW}$, a voltage deviation of 0.1171 p.u., and a $\mathrm{VSI}^{-1}$ value of 1.4367 p.u. It is assumed that the network has five opened switches and three connected DG units.

(a) Case 1: Simultaneous Optimization of Real Power Loss and Voltage Profile. In the first case, the NSSFS algorithm simultaneously optimized real power loss and voltage profile. Figure 6 depicts the Pareto optimal front generated by NSSFS.

Table 3 presents optimal results yielded by the NSSFS algorithm for the best individual objectives (best $P_{L}$ and best VD objectives) and the best compromise solution. According to Table 3, the NSSFS algorithm obtained the optimal opened switches: \{33-34-11-3128\}, optimal positions to connect three DGs at buses 7 , 25, and 17 with optimal sizes of $1.0995 \mathrm{MW}$, 1.5317 MW, and 1.0331 MW for the best compromise solution. It was shown that the real power loss and the voltage deviation were reduced to $57.2425 \mathrm{~kW}$ and 0.0022377 p.u., respectively. As a result, power loss and voltage deviation reductions were $71.75 \%$ and $98.09 \%$, respectively, compared to the initial case values.

(b) Case 2: Simultaneous Optimization of Real Power Loss and Voltage Stability Index. In Case 2, NSSFS simultaneously optimized the real power loss and voltage stability index, Figure 7 depicts the Pareto optimal front achieved by the NSSFS method for Case 2.

Table 4 represents optimal results for the best individual objectives (best $P_{L}$ and $\mathrm{VSI}^{-1}$ objectives) and the best tradeoff solution (best compromise solution) found by the NSSFS algorithm. According to the results, optimal opened switches for the best compromise solution were $\{7-14-9-30-27\}$, along with three DGs of sizes $1.4951 \mathrm{MW}, 1.0152 \mathrm{MW}$, and $0.5948 \mathrm{MW}$ were placed at buses 25,18 , and 12 , respectively, for the best compromise solution. It pointed out that NSSFS reduced the real power loss to $56.9306 \mathrm{~kW}$, corresponding to a power loss reduction of $71.91 \%$. Simultaneously, NSSFS also reduced the $\mathrm{VSI}^{-1}$ value to 1.0632 p.u., corresponding to a reduction of $25.99 \%$ in the $\mathrm{VSI}^{-1}$ value.

(c) Case 3: Simultaneous Optimization of Real Power Loss, Voltage Profile, and Voltage Stability Index. In Case 3, the NSSFS algorithm simultaneously optimized the three objectives, including real power loss, voltage profile, and voltage stability index. Figure 8 illustrates the Pareto optimal front yielded by the NSSFS, which clearly shows the relationships among the three objectives.

Table 5 presents the optimal results for the best individual objectives (best $P_{L}, \mathrm{VD}$, and $\mathrm{VSI}^{-1}$ objectives) and the best compromise solution achieved by NSSFS for Case 3. As observed in Table 5, NSSFS obtained the optimal opened switches: $\{33-34-11-31-$ $28\}$. The DGs were installed to the optimal positions at buses 17, 29, and 7 with optimal sizes of 1.0831 MW, 1.4465 MW, and 1.1621 MW for the best compromise solution. Accordingly, the real power loss, voltage deviation, and $\mathrm{VSI}^{-1}$ values were reduced to $61.0672 \mathrm{~kW}, 0.0015971$ p.u, and 1.0573 p.u., respectively, corresponding to a power loss reduction of $69.87 \%$, a voltage profile reduction of $98.64 \%$, along with a reduction of $26.41 \%$ in $\mathrm{VSI}^{-1}$ from the base case values. 
TABLE 2: Statistical results of MOO algorithms based on GD, SP, and $\Delta$ metrics.

\begin{tabular}{|c|c|c|c|c|c|c|}
\hline \multirow{2}{*}{ Algorithms } & \multicolumn{2}{|c|}{ GD } & \multicolumn{2}{|c|}{ SP } & \multicolumn{2}{|c|}{$\Delta$} \\
\hline & Average & SD & Average & Average & SD & Average \\
\hline \multicolumn{7}{|l|}{ ZDT1 } \\
\hline NSSFS & $3.4924 E-04$ & $1.0782 E-04$ & $6.8976 E-03$ & $1.2153 E-03$ & 0.4764 & $3.5008 E-02$ \\
\hline NSGA-II & $1.7379 E-01$ & $8.0688 E-03$ & $3.8312 \mathrm{E}-02$ & $1.1318 E-02$ & 0.7582 & $3.5549 E-02$ \\
\hline MOPSO & $9.8342 E-02$ & $2.0910 E-02$ & $3.1625 E-02$ & $1.8863 E-02$ & 0.8594 & $4.5138 E-02$ \\
\hline MOMVO & $1.8729 E-02$ & $5.7416 E-03$ & $1.3706 \mathrm{E}-02$ & $3.9621 E-03$ & 0.9378 & $5.6372 E-02$ \\
\hline \multicolumn{7}{|l|}{ ZDT2 } \\
\hline NSSFS & $3.5047 E-04$ & $1.1329 E-04$ & $6.8281 E-03$ & $9.7193 E-04$ & 0.4667 & $3.6285 E-02$ \\
\hline NSGA-II & $2.4868 E-01$ & $5.8042 E-03$ & $4.2047 \mathrm{E}-02$ & $8.3046 E-03$ & 0.8307 & $4.0518 E-02$ \\
\hline MOPSO & $1.2987 E-01$ & $7.6977 E-02$ & $1.6041 \mathrm{E}-02$ & $2.6287 E-02$ & 0.9331 & $7.9101 E-02$ \\
\hline MOMVO & $2.5871 E-02$ & $9.0512 E-03$ & $3.4432 \mathrm{E}-02$ & $3.1352 E-02$ & 1.0357 & $5.0408 E-02$ \\
\hline \multicolumn{7}{|l|}{ ZDT3 } \\
\hline NSSFS & $4.0931 \mathrm{E}-04$ & $1.2244 \mathrm{E}-04$ & $7.9861 \mathrm{E}-03$ & $1.3756 \mathrm{E}-03$ & 0.6816 & $2.1543 \mathrm{E}-02$ \\
\hline NSGA-II & $1.5827 E-01$ & $9.4133 E-03$ & $3.6036 E-02$ & $9.7661 E-03$ & 0.7517 & $3.2576 E-02$ \\
\hline MOPSO & $1.1522 E-01$ & $2.7497 E-02$ & $3.4803 E-02$ & $1.3565 E-02$ & 0.8821 & $3.2618 E-02$ \\
\hline MOMVO & $1.5197 E-02$ & $7.8896 E-03$ & $2.7471 E-02$ & $7.1647 E-03$ & 0.9585 & $7.0493 E-02$ \\
\hline \multicolumn{7}{|l|}{ ZDT6 } \\
\hline NSSFS & $8.7560 E-04$ & $8.6079 E-05$ & $7.3959 E-03$ & $1.7995 E-03$ & 0.4616 & $2.5479 E-02$ \\
\hline NSGA-II & $5.3467 E-01$ & $5.3694 E-03$ & $5.3084 E-02$ & $1.6892 E-02$ & 0.8650 & $4.0281 E-02$ \\
\hline MOPSO & $6.7379 E-01$ & $2.7311 E-01$ & $2.7517 E-01$ & $1.9551 E-01$ & 1.0073 & $7.3178 E-02$ \\
\hline MOMVO & $1.5929 E-01$ & $9.1610 E-02$ & $1.8520 E-01$ & $1.3259 E-01$ & 1.0757 & $1.1420 E-01$ \\
\hline \multicolumn{7}{|l|}{ KUR } \\
\hline NSSFS & $1.7650 E-03$ & $1.9376 E-04$ & $8.6902 E-02$ & $1.9891 E-02$ & 0.5128 & $3.0131 E-02$ \\
\hline NSGA-II & $5.4968 E-03$ & $1.6607 E-03$ & $9.3080 E-02$ & $3.4372 E-02$ & 0.6723 & $4.8597 E-02$ \\
\hline MOPSO & $1.2043 E-02$ & $4.3226 E-03$ & $1.4447 E-01$ & $6.3492 E-02$ & 0.6902 & $1.1214 E-01$ \\
\hline MOMVO & $4.0129 E-03$ & $6.9309 E-04$ & $1.1576 E-01$ & $3.0256 E-02$ & 0.9266 & $6.4492 E-02$ \\
\hline \multicolumn{7}{|l|}{ LAU } \\
\hline NSSFS & $3.1322 E-03$ & $9.0650 E-05$ & $3.0497 E-02$ & $5.4926 E-03$ & 0.5606 & $5.9600 E-02$ \\
\hline NSGA-II & $5.0307 E-03$ & $5.8411 E-03$ & $4.1044 E-02$ & $8.3596 E-02$ & 1.3172 & $9.8168 E-02$ \\
\hline MOPSO & $3.1971 E-03$ & $2.3021 E-04$ & $3.6111 E-02$ & $1.3438 E-02$ & 0.4670 & $3.9501 \mathrm{E}-02$ \\
\hline MOMVO & $3.1846 E-03$ & $1.6359 E-04$ & $4.5949 E-02$ & $6.5992 E-03$ & 0.7591 & $4.4917 E-02$ \\
\hline \multicolumn{7}{|l|}{ MUR } \\
\hline NSSFS & $4.7949 E-04$ & $1.2667 E-05$ & $2.1203 E-02$ & $2.4248 E-03$ & 0.4601 & $4.3902 E-02$ \\
\hline NSGA-II & $6.3278 E-04$ & $1.6361 E-03$ & $2.2789 E-02$ & $1.2927 E-02$ & 0.4193 & $4.3942 E-02$ \\
\hline MOPSO & $1.7177 E-03$ & $6.9557 E-04$ & $1.8243 E-02$ & $3.2174 E-03$ & 0.4374 & $4.3754 E-02$ \\
\hline MOMVO & $1.4261 E-03$ & $2.9123 E-03$ & $4.4461 E-02$ & $2.1436 E-02$ & 1.3302 & $4.3489 \mathrm{E}-02$ \\
\hline \multicolumn{7}{|l|}{$\overline{\mathrm{POL}}$} \\
\hline NSSFS & $1.7230 E-03$ & $2.5106 E-04$ & 0.1056 & $1.1257 E-02$ & 0.9561 & $1.9904 E-02$ \\
\hline NSGA-II & $2.0110 E-02$ & $2.0514 E-02$ & 0.1199 & $4.0498 E-02$ & 0.9841 & $3.1525 E-02$ \\
\hline MOPSO & $1.8498 E-02$ & $1.5407 E-02$ & 0.1947 & $1.2153 E-01$ & 1.0065 & $2.9527 E-02$ \\
\hline MOMVO & $1.8770 E-02$ & $3.1453 E-02$ & 0.1951 & $5.8515 E-02$ & 1.4341 & $3.4362 E-02$ \\
\hline
\end{tabular}

Given the results obtained from this case, the consideration of DNR-DG indicated a remarkable enhancement in all three objective functions. This was further confirmed by observing Figures 9 and 10, which portray the voltage profiles and VSI values of the system for Cases 1-3, respectively. The voltage profiles and VSI of the system for Case 3 provided better improvement than Cases 1-2. Therefore, it could be stated that the optimal result for the best compromise solution satisfied all the objectives at their maximum extents simultaneously.

5.2.2. 69-Bus RDN. The NSSFS method was applied to the 69-bus RDN to validate its scalability. Detailed data can be derived from Ref. [53]. For the initial case, real power loss, voltage deviation, and VSI-1 value of the system are $225 \mathrm{~kW}$, 0.0993 p.u., and 1.4614 p.u., respectively. The system has five opened switches and three installed DG units.

(a) Case 4: Simultaneous Optimization of Real Power Loss and Voltage Profile. The NSSFS algorithm was applied to optimize the real power loss and voltage deviation simultaneously. Figure 11 depicts the Pareto optimal front found by NSSFS for Case 4 of the 69-bus network.

Table 6 gives optimal results for the best individual objectives and the best compromise solution yielded by NSSFS. The best compromise solution of the NSSFS algorithm obtained the optimal opened 


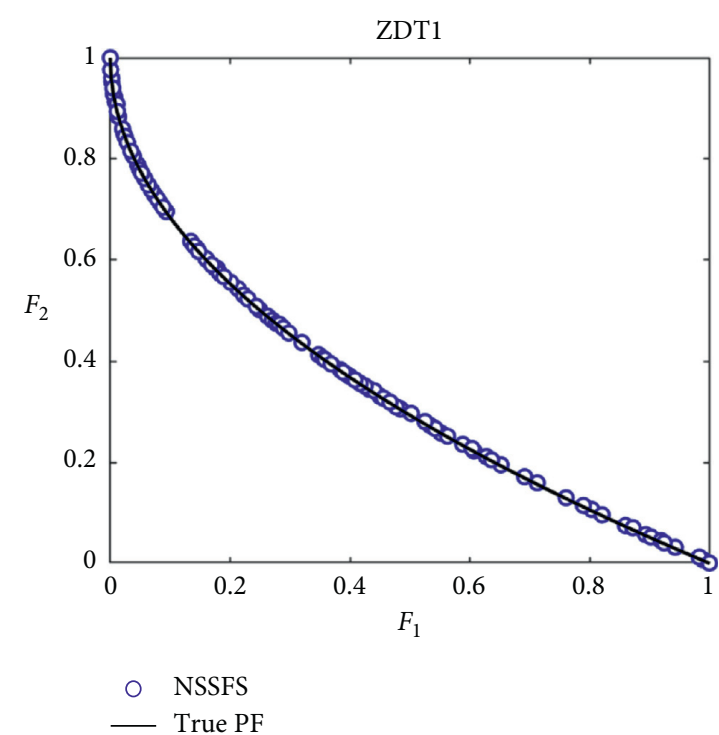

(a)

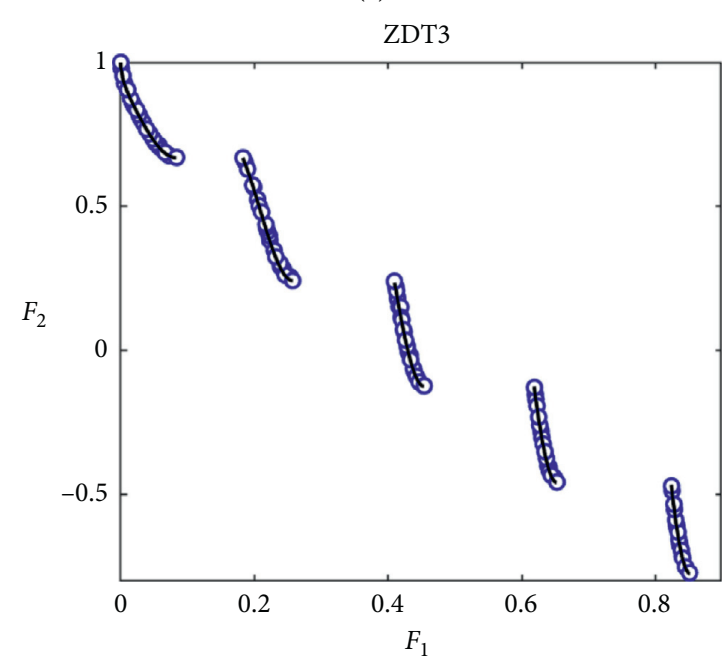

O NSSFS

_ True PF

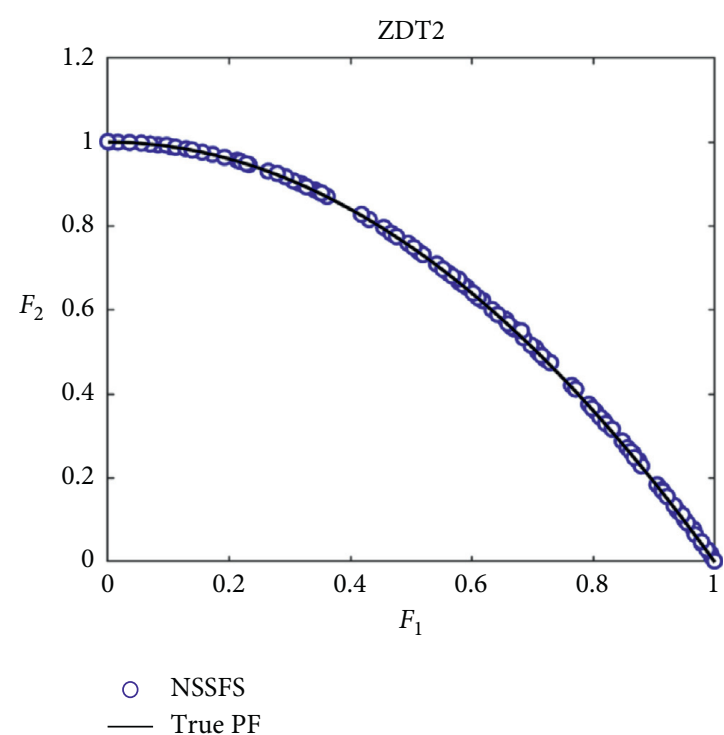

(b)

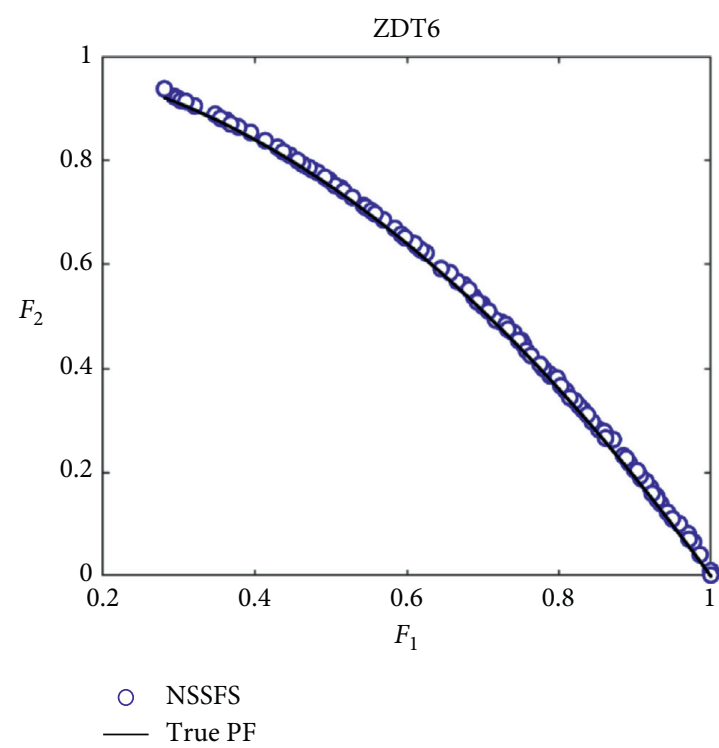

(d)

Figure 4: Pareto optimal fronts generated by NSSFS for test functions. (a) ZDT1, (b) ZDT2, (c) ZDT3, and (d) ZDT6.

switches: $\{69-18-14-58-61\}$, where three DG were installed to the system at buses 64,61 , and 12 with optimal sizes of $0.4564 \mathrm{MW}, 1.7811 \mathrm{MW}$, and $0.8298 \mathrm{MW}$, respectively. Accordingly, NSSFS simultaneously reduced the real power loss to $41.6990 \mathrm{~kW}$ (81.47\% power loss reduction), and the voltage deviation to 0.00095180 p.u. (99.04\% voltage deviation reduction) in comparison to the initial case values.

(b) Case 5. Simultaneous Optimization of Real Power Loss and Voltage Stability Index. The NSSFS optimized the real power loss and voltage stability index simultaneously. Figure 12 depicts the Pareto front yielded by NSSFS for Case 5 .

Table 7 shows the best individual objectives and the best trade-off solutions obtained by NSSFS for Case 5. From Table 7, NSSFS obtained opened switches: \{69-70-14-58-63\} for the best compromise solution. The optimal positions of DGs were also found at buses 61, 27, and 11 with the optimal sizes of $1.9194 \mathrm{MW}, 0.5819 \mathrm{MW}$, and $0.5508 \mathrm{MW}$, respectively. After the implementation of DNR-DG, NSSFS offered a real power loss of $40.0405 \mathrm{~kW}$ and a $\mathrm{VSI}^{-1}$ value of 1.0434 p.u. In other words, the power loss 


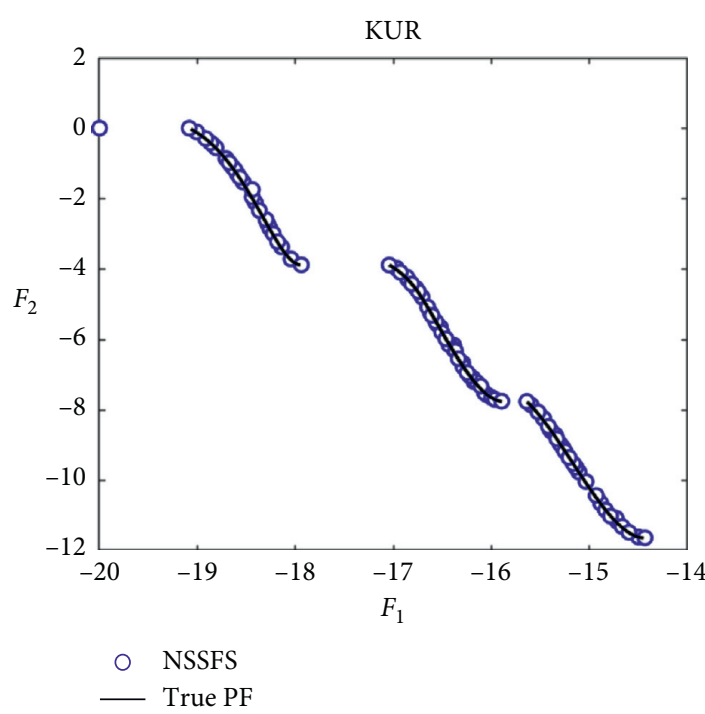

(a)

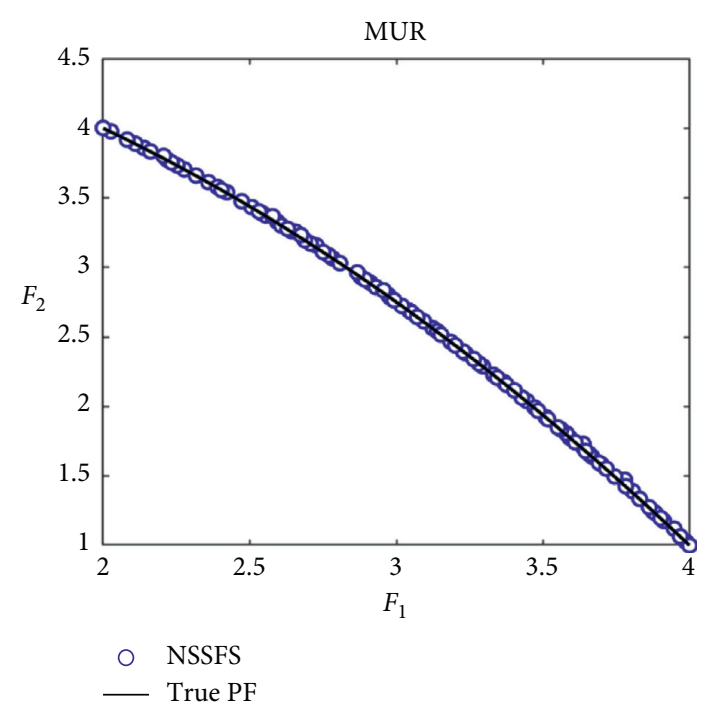

(c)

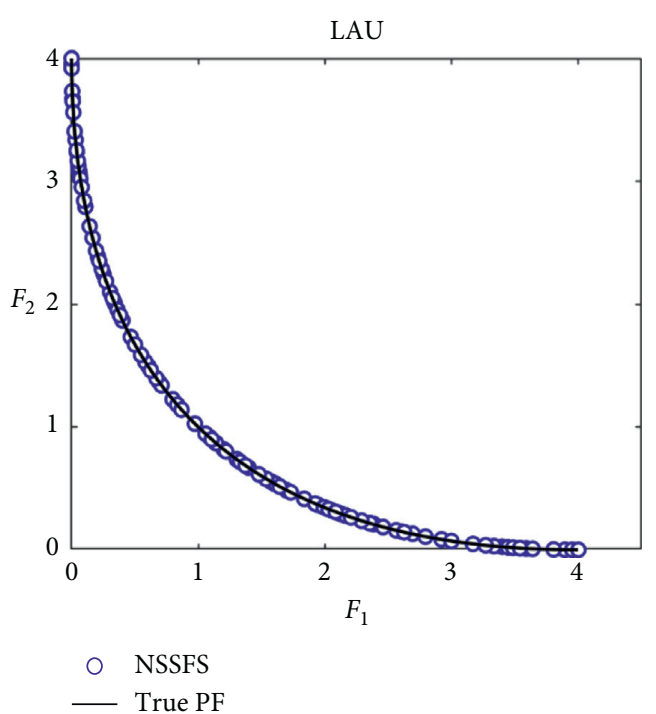

(b)

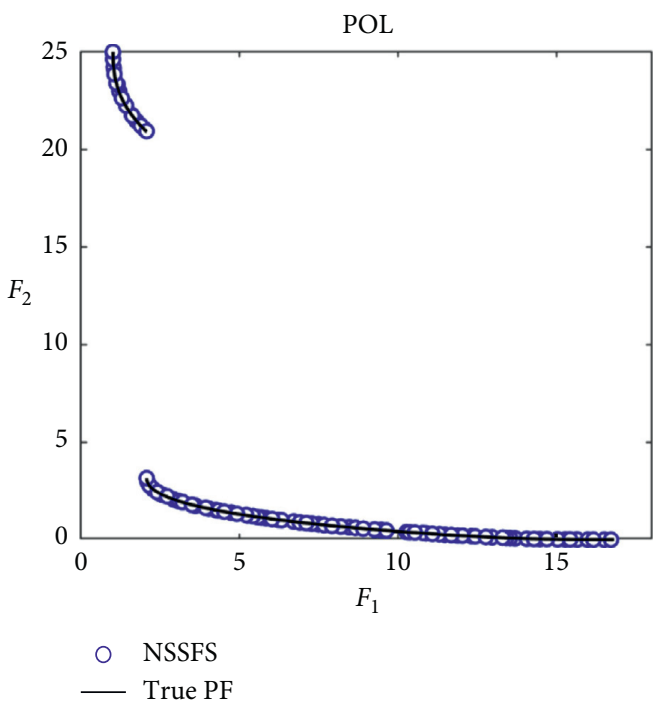

(d)

FIgURE 5: Pareto optimal fronts generated by NSSFS for test functions. (a) KUR, (b) LAU, (c) MUR, and (d) POL.

reduction and $\mathrm{VSI}^{-1}$ reduction for this case were $82.20 \%$ and $28.60 \%$, respectively.

(c) Case 6: Simultaneous Optimization of Real Power Loss, Voltage Profile, and Voltage Stability Index. The power loss, voltage profile, and voltage stability index were simultaneously optimized by the NSSFS method. Figure 13 depicts the Pareto front generated by NSSFS.

The optimal results obtained by NSSFS are presented in Table 8. As observed in Table 8, the best compromise solution found by NSSFS yielded the optimal opened switches: $\{69-17-14-57-61\}$ and obtained the optimal positions at buses 12,62 , and 61 corresponding to optimal sizes of $0.9516 \mathrm{MW}, 0.5176 \mathrm{MW}$, and $1.9986 \mathrm{MW}$. According to the results, NSSFS achieved a real power loss of $49.7107 \mathrm{~kW}$, voltage deviation of
0.00068338 p.u., and $\mathrm{VSI}^{-1}$ value of 1.0413 p.u., corresponding to a power loss reduction of $77.91 \%$, a voltage deviation reduction of $99.31 \%$, and $\mathrm{VSI}^{-1}$ reduction of $28.75 \%$, respectively, compared to initial case values. It could be indicated that the implementation of DNRDG provided a remarkable enhancement in all three objectives. To further confirm the above claim, Figures 14 and 15 depict the voltage profiles and VSI value of the RDN for Cases 4-6, respectively, where optimal results for Case 6 showed a better enhancement than Cases $4-5$ in voltage profiles and VSI.

5.3. Statistical Comparison and Analysis. To assess the applicability of NSSFS, the NSGA-II, MOPSO, and MOMVO algorithms were also executed to the MODNR-DG problem as result comparisons. For each case study, all multiobjective 


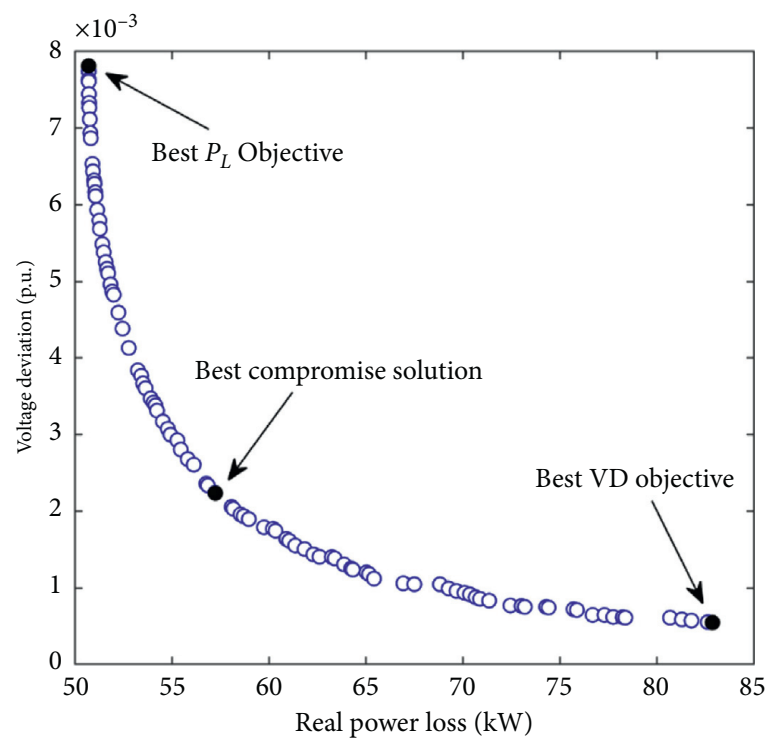

Figure 6: Pareto optimal front generated by NSSFS for Case 1.

Table 3: Optimal results of best individual objectives and best compromise solution for Case 1.

\begin{tabular}{lccr}
\hline Parameter & Best $P_{L}$ objective & Best VD objective & Best compromise solution \\
\hline Opened switches & $33-34-11-31-28$ & $33-12-11-31-28$ & $33-34-11-31-28$ \\
\hline \multirow{2}{*}{ DG } & $17(0.9583)$ & $9(1.1693)$ & $7(1.0995)$ \\
& $25(1.2785)$ & $30(1.7450)$ & $25(1.5317)$ \\
\hline$P_{L}(\mathrm{~kW})$ & $7(0.7528)$ & $18(0.8007)$ & $17(1.0331)$ \\
\hline VD (p.u.) & 50.7176 & 82.8434 & 57.2425 \\
\hline
\end{tabular}

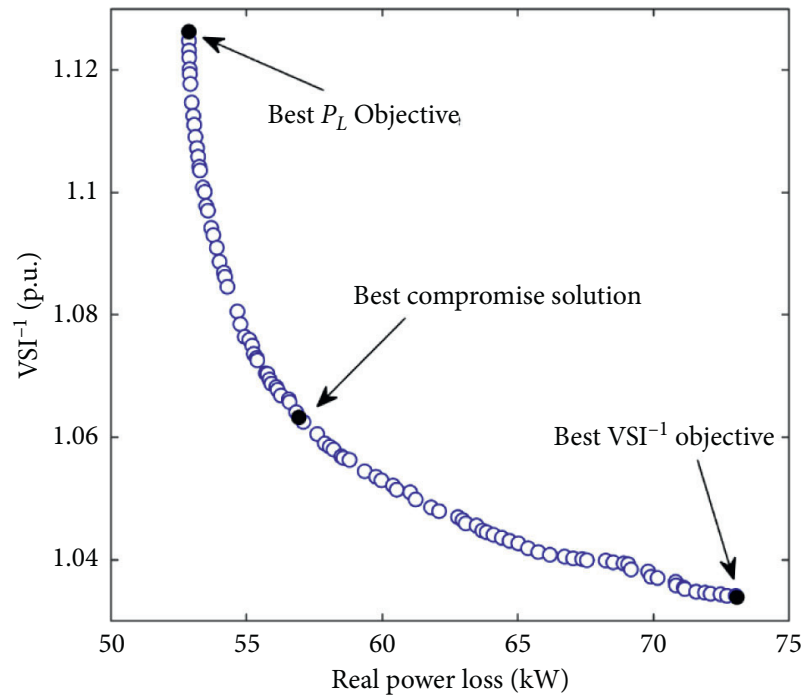

FIgURe 7: Pareto optimal front generated by NSSFS for Case 2.

algorithms were carried out thirty runs independently with an equal NFEs. The parameter settings of the multiobjective algorithms were chosen, as seen in Table 1. Optimization results were compared based on SP and HV metrics. Since the HV metric defined in equation (33) is not available for problems having more than two objective functions, the optimal results for Cases 3 and 6 were only compared based on the SP metric.

Table 9 presents the statistical results of performance metrics (SP and HV metrics) for NSSFS, NSGA-II, MOPSO, and MOMVO algorithms for the 33-bus RDN. Considering the SP metrics from Table 9, NSSFS showed superior performance to preserve an appropriate distribution of nondominated solutions. Similarly, NSSFS surpassed NSGA-II, MOPSO, and MOMVO for HV metrics. Hence, it could be inferred that the NSSFS offered well-distributed Pareto fronts for all case studies of the 33-bus RDN.

Table 10 presents the statistical results of SP and HV metrics yielded by NSSFS, NSGA-II, MOPSO, and MOMVO for the 69-bus RDN. Based on Table 10, the average SP value found by NSSFS ranked first. Hence, NSSFS yielded more consistently distributed Pareto fronts than other algorithms. Also, the NSSFS algorithm also obtained the best outcomes for HV metrics in Table 10. It should be mentioned that the NSSFS and MOMVO produced competitive results in terms of the HV metric, but the SP value for NSSFS was much better than the value for MOMVO. Therefore, NSSFS obtained a broad range of solutions with a uniform spread for three case studies of the 69-bus RDN. 
TABLE 4: Optimal results of best individual objectives and best compromise solution for Case 2 .

\begin{tabular}{lccc}
\hline Parameter & Best $P_{L}$ objective & Best VSI ${ }^{-1}$ objective & Best compromise solution \\
\hline Opened switches & $7-14-9-30-27$ & $7-14-9-30-25$ & $7-14-9-30-27$ \\
\hline \multirow{2}{*}{ DG } & $25(1.2906)$ & $29(1.8323)$ & $25(1.4951)$ \\
& $18(0.7954)$ & $17(1.2013)$ & $18(1.0152)$ \\
\hline$P_{L}(\mathrm{~kW})$ & $12(0.5709)$ & $12(0.6782)$ & $12(0.5948)$ \\
\hline $\mathrm{VSI}^{-1}$ (p.u.) & 52.8798 & 73.0682 & 56.9306 \\
\hline
\end{tabular}

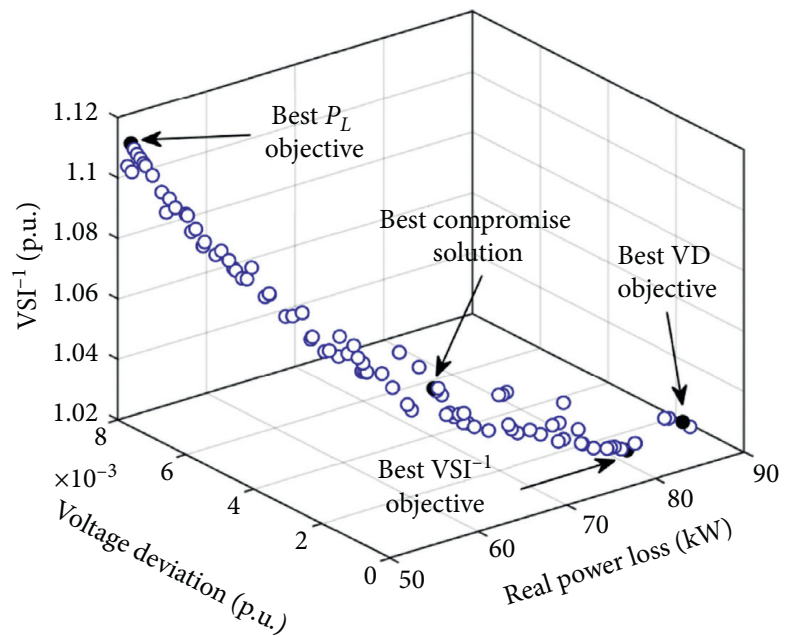

Figure 8: Pareto optimal front generated by NSSFS for Case 3.

Table 5: Optimal results of best individual objectives and best compromise solution for Case 3 .

\begin{tabular}{|c|c|c|c|c|}
\hline Parameter & Best $P_{L}$ objective & Best VD objective & Best $\mathrm{VSI}^{-1}$ objective & Best compromise solution \\
\hline Opened switches & $33-34-11-31-28$ & $33-13-35-30-28$ & $33-13-35-30-28$ & $33-34-11-31-28$ \\
\hline DG & $\begin{array}{c}17(0.7529) \\
25(1.2791) \\
7(0.9588)\end{array}$ & $\begin{array}{c}18(1.0501) \\
30(1.4724) \\
9(1.1919)\end{array}$ & $\begin{array}{c}18(1.0482) \\
29(1.5676) \\
9(1.0986)\end{array}$ & $\begin{array}{c}17(1.0831) \\
29(1.4465) \\
7(1.1621) \\
\end{array}$ \\
\hline$P_{L}(\mathrm{~kW})$ & 50.7176 & 84.9654 & 79.0634 & 61.0672 \\
\hline VD (p.u.) & 0.0078071 & 0.00049619 & 0.00059821 & 0.0015971 \\
\hline $\mathrm{VSI}^{-1}$ (p.u.) & 1.1119 & 1.0315 & 1.0267 & 1.0573 \\
\hline
\end{tabular}

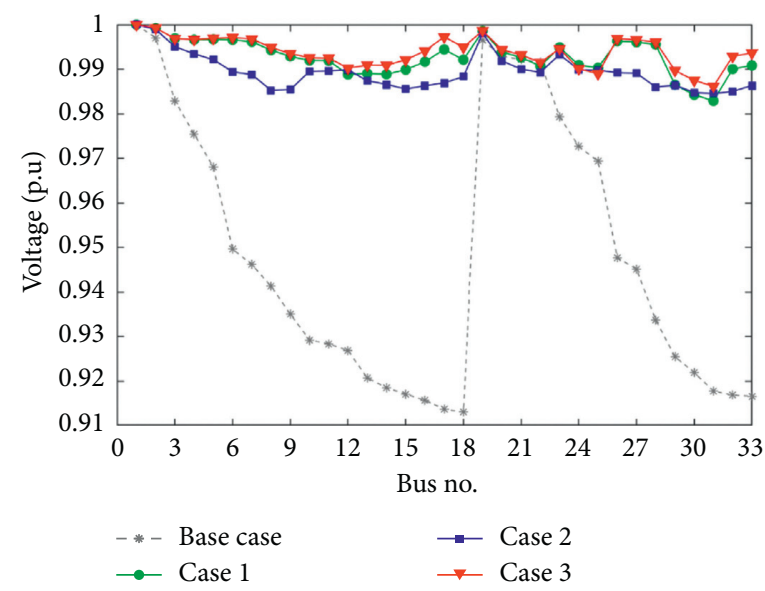

Figure 9: Voltage profiles of the systems for Cases 1-3. 


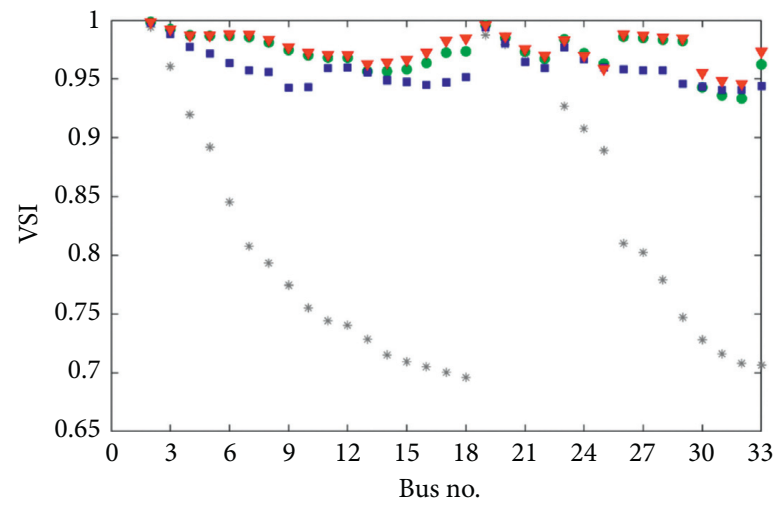
* Base case
- Case 2
- Case 1
$\checkmark$ Case 3

Figure 10: VSI at buses of the systems for Cases 1-3.

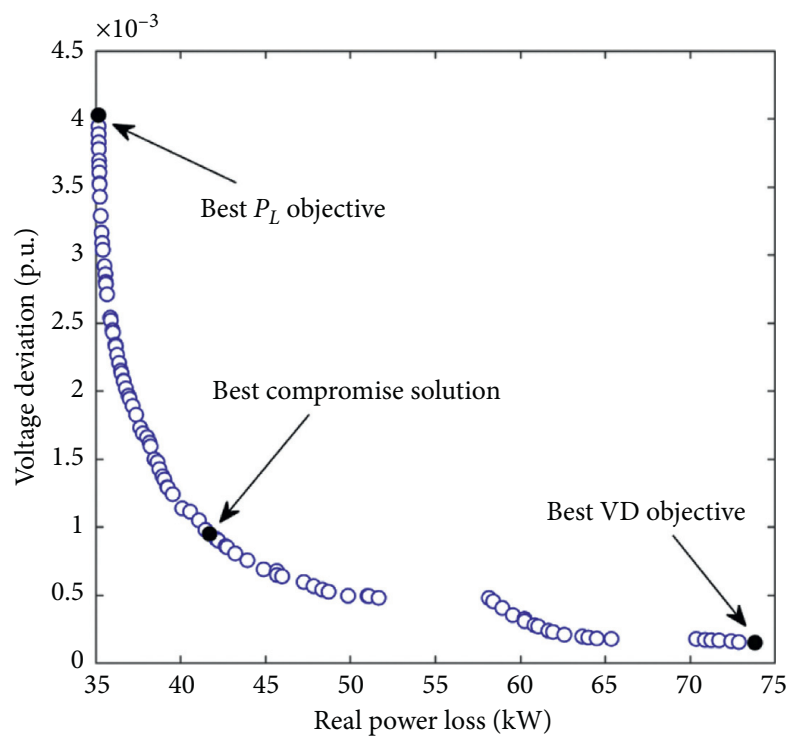

Figure 11: Pareto optimal front generated by NSSFS for Case 4.

Table 6: Optimal results of best individual objectives and best compromise solution for Case 4.

\begin{tabular}{lccc}
\hline Parameter & Best $P_{L}$ objective & Best VD objective & Best compromise solution \\
\hline Opened switches & $69-70-14-58-61$ & $69-15-14-72-61$ & $69-18-14-58-61$ \\
\hline \multirow{2}{*}{ DG } & $64(0.4905)$ & $65(0.5903)$ & $64(0.4564)$ \\
& $61(1.4339)$ & $61(1.8305)$ & $61(1.7811)$ \\
\hline$P_{L}(\mathrm{~kW})$ & $11(0.5377)$ & $12(1.0447)$ & $12(0.8298)$ \\
\hline VD (p.u.) & 35.1624 & 73.8018 & 41.6990 \\
\hline
\end{tabular}




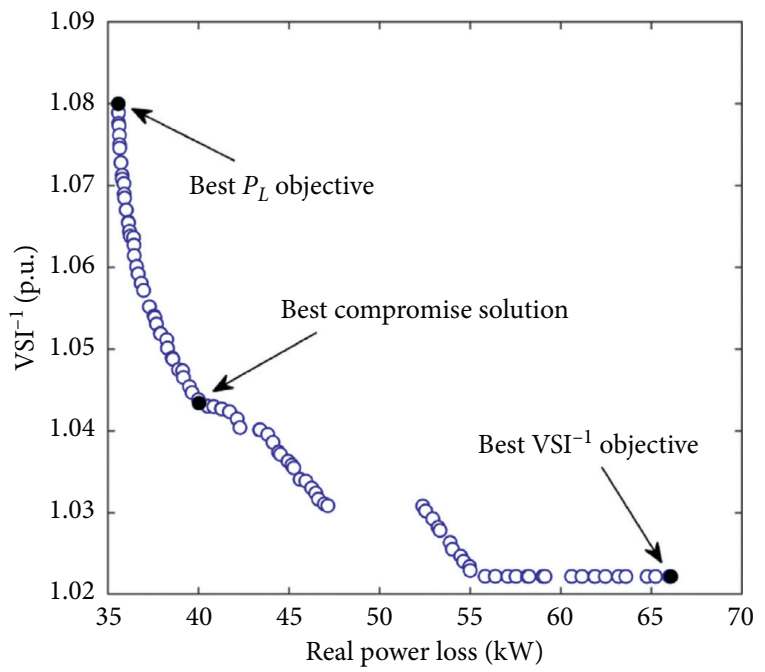

Figure 12: Pareto optimal front generated by NSSFS for Case 5.

TABLE 7: Optimal results of best individual objectives and best compromise solution for Case 5 .

\begin{tabular}{lccr}
\hline Parameter & Best $P_{L}$ objective & Best VSI ${ }^{-1}$ objective & Best compromise solution \\
\hline Opened switches & $69-70-14-57-63$ & $9-14-12-72-63$ & $69-70-14-58-63$ \\
\hline \multirow{2}{*}{ DG } & $61(1.4668)$ & $61(1.9994)$ & $61(1.9194)$ \\
& $27(0.5329)$ & $25(0.7486)$ & $27(0.5819)$ \\
\hline$P_{L}(\mathrm{~kW})$ & $11(0.5374)$ & $11(1.0539)$ & $11(0.5508)$ \\
\hline $\mathrm{VSI}^{-1}$ (p.u.) & 35.5914 & 66.0568 & 40.0405 \\
\hline
\end{tabular}

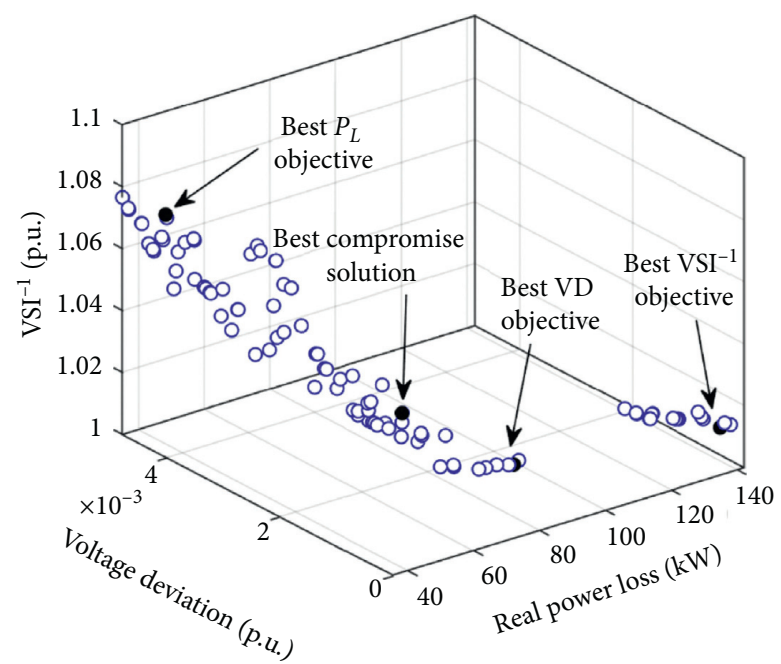

FIGURE 13: Pareto optimal front generated by NSSFS for Case 6. 
TABLE 8: Optimal results of best individual objectives and best compromise solution for Case 6.

\begin{tabular}{lcccr}
\hline Parameter & Best $P_{L}$ objective & Best VD objective & Best VSI ${ }^{-1}$ objective & Best compromise solution \\
\hline Opened switches & $10-70-13-58-62$ & $10-18-12-72-63$ & $35-13-12-72-62$ & $69-17-14-57-61$ \\
\hline \multirow{2}{*}{ DG } & $61(1.4671)$ & $61(1.8760)$ & $61(1.8683)$ & $12(0.9516)$ \\
& $27(0.4750)$ & $26(0.7332)$ & $27(0.6420)$ & $62(0.5176)$ \\
\hline$P_{L}(\mathrm{~kW})$ & $43(0.6626)$ & $44(1.1917)$ & $44(1.2114)$ & $61(1.9986)$ \\
\hline VD (p.u.) & 35.1624 & 73.9341 & 139.4720 & 49.7107 \\
\hline VSI $^{-1}$ (p.u.) & 0.0040278 & 0.00011574 & 0.00027253 & 0.00068338 \\
\hline
\end{tabular}

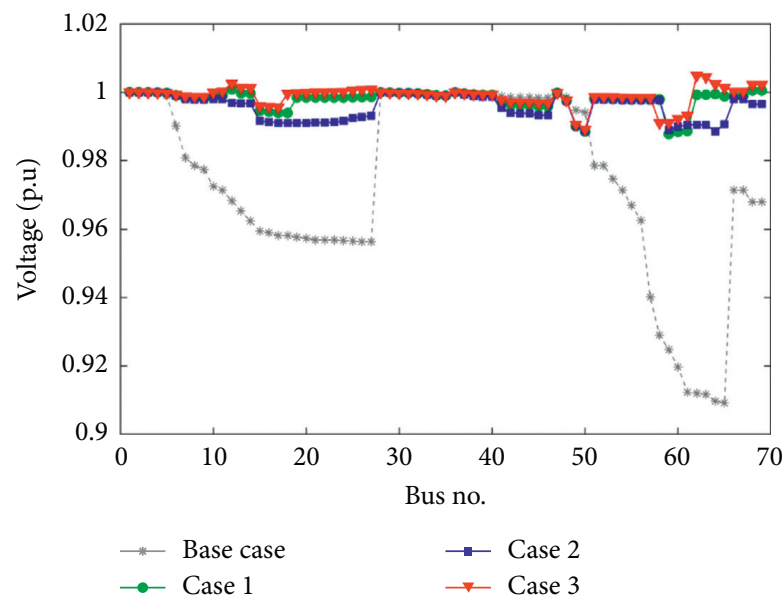

Figure 14: Voltage profiles of the systems for Cases 4-6.

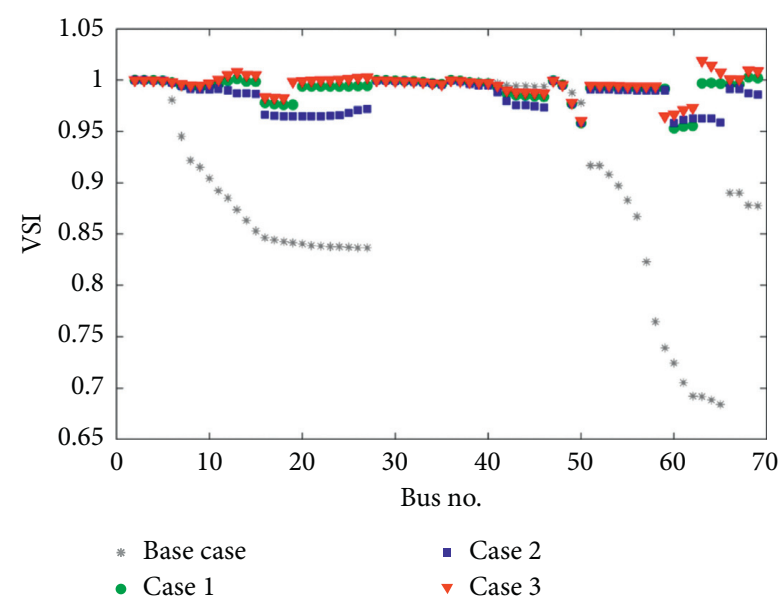

FIGURE 15: VSI at buses of the systems for Cases 4-6. 
TABLE 9: Comparative results of MOO algorithms based on HV and SP metrics for 33-bus RDN.

\begin{tabular}{lcccc}
\hline \multirow{2}{*}{ Algorithm } & \multicolumn{2}{c}{ SP } & \multicolumn{2}{c}{$\mathrm{HV}$} \\
& Average & SD & Average & SD \\
\hline Case 1 & & & & \\
NSSFS & 0.2236 & 0.0619 & 0.9102 & 0.0272 \\
NSGA-II & 2.5775 & 2.6177 & 0.7123 & 0.0174 \\
MOPSO & 0.3631 & 0.3969 & 0.8459 & 0.0344 \\
MOMVO & 0.3489 & 0.2223 & 0.8888 & 0.0427 \\
\hline Case 2 & & & & \\
NSSFS & 0.1971 & 0.2947 & 30.4346 & 0.3273 \\
NSGA-II & 4.0279 & 3.3422 & 26.1267 & 0.5894 \\
MOPSO & 0.5038 & 0.5036 & 28.3043 & 1.1903 \\
MOMVO & 0.2454 & 0.1853 & 29.6101 & 0.9595 \\
\hline Case 3 & & & & \\
NSSFS & 0.3333 & 0.0963 & - & - \\
NSGA-II & 4.0933 & 2.4830 & - & - \\
MOPSO & 1.5299 & 1.6161 & - & - \\
MOMVO & 0.8103 & 0.7418 & - & - \\
\hline
\end{tabular}

TABLE 10: Comparative results of MOO algorithms based on HV and SP metrics for 69-bus RDN.

\begin{tabular}{lcccc}
\hline \multirow{2}{*}{ Algorithm } & \multicolumn{2}{c}{ SP } & \multicolumn{2}{c}{$\mathrm{HV}$} \\
& Average & SD & Average & SD \\
\hline Case 4 & & & & \\
NSSFS & 0.1992 & 0.0424 & 1.2132 & 0.0278 \\
NSGA-II & 1.8794 & 1.1493 & 1.1001 & 0.0147 \\
MOPSO & 0.5698 & 1.4181 & 1.1348 & 0.0460 \\
MOMVO & 0.3657 & 0.3647 & 1.2062 & 0.0311 \\
\hline Case 5 & & & & \\
NSSFS & 0.2167 & 0.0746 & 39.4501 & 0.3243 \\
NSGA-II & 3.0382 & 2.1226 & 37.2145 & 0.4841 \\
MOPSO & 1.3226 & 2.6240 & 37.1660 & 1.2296 \\
MOMVO & 0.3062 & 0.1282 & 39.3065 & 0.7039 \\
\hline Case 6 & & & & \\
NSSFS & 0.4311 & 0.1028 & - & - \\
NSGA-II & 2.6718 & 1.6744 & - & - \\
MOPSO & 3.8535 & 4.2420 & - & - \\
MOMVO & 0.7742 & 0.8450 & - & - \\
\hline
\end{tabular}

\section{Conclusion}

This study presented a new multiobjective version of the SFS method called NSSFS for dealing with the MODNR-DG problem. The NSSFS integrated the fast nondominated sorting and crowding distance techniques to define the nondominated ranks of obtained solutions. Also, the selection mechanism is used to maintain potential candidate solutions for the next generation. NSSFS was first tested with eight multiobjective benchmark functions with different types and dimensions. Comparative results based on performance metrics (GD, SP, and $\Delta$ metrics) showed that the NSSFS algorithm achieved better performance than NSGAII, MOPSO, and MOMVO for solution quality. After validation, the NSSFS method was effectively applied to the MODNR-DG problem in RDNs. The real power loss, voltage profile, and voltage stability index were considered to formulate the MODNR-DG problem. The performance of NSSFS was demonstrated on the 33-bus and 69-bus RDNs with six case studies. The obtained results showed that the implementation of DNR-DG pointedly enhanced the quality of the system in RDNs. This can be seen through Case 6 of the 69-bus network, where the real power loss, voltage deviation, and $\mathrm{VSI}^{-1}$ value were reduced by $77.91 \%, 99.31 \%$, and $28.75 \%$, respectively. The comparison results indicated that the NSSFS algorithm yielded better solution quality than other multiobjective algorithms based on SP and HV metrics. Therefore, NSSFS proved to be an auspicious technique to deal with the MODNR-DG problem in RDNs.

\section{Data Availability}

No data were used to support the findings of this study..

\section{Conflicts of Interest}

The authors declare that they have no conflicts of interest.

\section{Acknowledgments}

This research was funded by the Ho Chi Minh City University of Technology, VNU-HCM, under Grant number T-ĐĐТ-2020-30.

\section{References}

[1] S. Gopiya Naik, D. K. Khatod, and M. P. Sharma, "Optimal allocation of combined DG and capacitor for real power loss minimization in distribution networks," International Journal of Electrical Power \& Energy Systems, vol. 53, pp. 967-973, 2013.

[2] M. R. Islam, H. Lu, M. J. Hossain, and L. Li, "Mitigating unbalance using distributed network reconfiguration techniques in distributed power generation grids with services for electric vehicles: a review," Journal of Cleaner Production, vol. 239, p. 117932, 2019.

[3] J. Zhu, X. Xiong, J. Zhang, G. Shen, Q. Xu, and Y. Xue, “A rule based comprehensive approach for reconfiguration of electrical distribution network," Electric Power Systems Research, vol. 79, no. 2, pp. 311-315, 2009.

[4] T. Niknam, E. A. Farsani, and M. Nayeripour, "An efficient multi-objective modified shuffled frog leaping algorithm for distribution feeder reconfiguration problem," European Transactions on Electrical Power, vol. 21, no. 1, pp. 721-739, 2011.

[5] M. R. Andervazh, J. Olamaei, and M. R. Haghifam, "Adaptive multi-objective distribution network reconfiguration using multi-objective discrete particles swarm optimisation algorithm and graph theory," IET Generation, Transmission \& Distribution, vol. 7, no. 12, pp. 1367-1382, 2013.

[6] M. Sedighizadeh, S. Ahmadi, and M. Sarvi, "An efficient hybrid big bang-big crunch algorithm for multi-objective reconfiguration of balanced and unbalanced distribution systems in fuzzy framework," Electric Power Components and Systems, vol. 41, no. 1, pp. 75-99, 2013.

[7] F.-Y. Hsu and M.-S. Tsai, "A non-dominated sorting evolutionary programming algorithm for multi-objectives power distribution system feeder reconfiguration problems," International Transactions on Electrical Energy Systems, vol. 23, no. 2, pp. 191-213, 2013. 
[8] H. D. Dehnavi and S. Esmaeili, "A new multiobjective fuzzy shuffled frog-leaping algorithm for optimal reconfiuration of radial distribution systems in the presence of reactive power compensators," Turkish Journal of Electrical Engineering \& Computer Sciences, vol. 21, pp. 864-881, 2013.

[9] M. R. Narimani, A. Azizi Vahed, R. Azizipanah-Abarghooee, and M. Javidsharifi, "Enhanced gravitational search algorithm for multi-objective distribution feeder reconfiguration considering reliability, loss and operational cost," IET Generation, Transmission \& Distribution, vol. 8, no. 1, pp. 55-69, 2014.

[10] A. Mohamed Imran and M. Kowsalya, "A new power system reconfiguration scheme for power loss minimization and voltage profile enhancement using fireworks algorithm," International Journal of Electrical Power \& Energy Systems, vol. 62, pp. 312-322, 2014.

[11] C. H. N. D. R. Barbosa, M. H. S. Mendes, and J. A. de Vasconcelos, "Robust feeder reconfiguration in radial distribution networks," International Journal of Electrical Power \& Energy Systems, vol. 54, pp. 619-630, 2014.

[12] N. Gupta, A. Swarnkar, and K. R. Niazi, "Distribution network reconfiguration for power quality and reliability improvement using genetic algorithms," International Journal of Electrical Power \& Energy Systems, vol. 54, pp. 664-671, 2014.

[13] A. Mazza, G. Chicco, and A. Russo, "Optimal multi-objective distribution system reconfiguration with multi criteria decision making-based solution ranking and enhanced genetic operators," International Journal of Electrical Power \& Energy Systems, vol. 54, pp. 255-267, 2014.

[14] Y. M. Shuaib, M. S. Kalavathi, and C. C. Asir Rajan, "Optimal reconfiguration in radial distribution system using gravitational search algorithm," Electric Power Components and Systems, vol. 42, no. 7, pp. 703-715, 2014.

[15] H. Shareef, A. A. Ibrahim, N. Salman, A. Mohamed, and W. Ling Ai, "Power quality and reliability enhancement in distribution systems via optimum network reconfiguration by using quantum firefly algorithm," International Journal of Electrical Power \& Energy Systems, vol. 58, pp. 160-169, 2014.

[16] D. Sudha Rani, N. Subrahmanyam, and M. Sydulu, "Multiobjective invasive weed optimization-an application to optimal network reconfiguration in radial distribution systems," International Journal of Electrical Power \& Energy Systems, vol. 73, pp. 932-942, 2015.

[17] M. Kaur and S. Ghosh, "Network reconfiguration of unbalanced distribution networks using fuzzy-firefly algorithm," Applied Soft Computing, vol. 49, pp. 868-886, 2016.

[18] T. T. Nguyen, T. T. Nguyen, A. V. Truong, Q. T. Nguyen, and T. A. Phung, "Multi-objective electric distribution network reconfiguration solution using runner-root algorithm," $A p$ plied Soft Computing, vol. 52, pp. 93-108, 2017.

[19] A. Mohamed Imran, M. Kowsalya, and D. P. Kothari, “A novel integration technique for optimal network reconfiguration and distributed generation placement in power distribution networks," International Journal of Electrical Power \& Energy Systems, vol. 63, pp. 461-472, 2014.

[20] R. S. Rao, K. Ravindra, K. Satish, and S. V. L. Narasimham, "Power loss minimization in distribution system using network reconfiguration in the presence of distributed generation," IEEE Transactions on Power Systems, vol. 28, no. 1, pp. 317-325, 2013.

[21] T. T. Nguyen, A. V. Truong, and T. A. Phung, "A novel method based on adaptive cuckoo search for optimal network reconfiguration and distributed generation allocation in distribution network," International Journal of Electrical Power \& Energy Systems, vol. 78, pp. 801-815, 2016.
[22] A. Onlam, D. Yodphet, R. Chatthaworn, C. Surawanitkun, A. Siritaratiwat, and P. Khunkitti, "Power loss minimization and voltage stability improvement in electrical distribution system via network reconfiguration and distributed generation placement using novel adaptive shuffled frogs leaping algorithm,” Energies, vol. 12, no. 3, p. 553, 2019.

[23] Z. Gong, Q. Chen, and K. Sun, "Novel methodology solving distribution network reconfiguration with DG placement," The Journal of Engineering, vol. 2019, no. 16, pp. 1668-1674, 2019.

[24] V. V. V. S. N. Murty and A. Kumar, "Optimal DG integration and network reconfiguration in microgrid system with realistic time varying load model using hybrid optimisation," IET Smart Grid, vol. 2, no. 2, pp. 192-202, 2019.

[25] H. B. Tolabi, M. H. Ali, S. B. M. Shahrin Bin, and M. Rizwan, "Novel hybrid fuzzy-bees algorithm for optimal feeder multiobjective reconfiguration by considering multiple-distributed generation," Energy, vol. 71, pp. 507-515, 2014.

[26] K. Muthukumar, "Integrated approach of network reconfiguration with distributed generation and shunt capacitors placement for power loss minimization in radial distribution networks," Applied Soft Computing, vol. 52, pp. 1262-1284, 2017.

[27] K. Buayai, "Optimal multi-type DGs placement in primary distribution system by NSGA-II," Research Journal of Applied Sciences, Engineering and Technology, vol. 4, pp. 3610-3617, 2012.

[28] R. Syahputra, I. Robandi, and M. Ashari, "Reconfiguration of distribution network with DG using fuzzy multi-objective method," in Proceedings of the 2012 International Conference on Innovation Management and Technology Research, pp. 316-321, Kuala Lumpur, Malaysia, 2012.

[29] A. R. Malekpour, T. Niknam, A. Pahwa, and A. Kavousi Fard, "Multi-objective stochastic distribution feeder reconfiguration in systems with wind power generators and fuel cells using the Point estimate method," IEEE Transactions on Power Systems, vol. 28, no. 2, pp. 1483-1492, 2013.

[30] V. K. Thunuguntla and S. K. Injeti, " $\varepsilon$-constraint multiobjective approach for optimal network reconfiguration and optimal allocation of DGs in radial distribution systems using the butterfly optimizer," International Transactions on Electrical Energy Systems, vol. 30, Article ID e12613, 2020.

[31] M. Chakravorty and D. Das, "Voltage stability analysis of radial distribution networks," International Journal of Electrical Power \& Energy Systems, vol. 23, no. 2, pp. 129-135, 2001.

[32] V. Savsani and M. A. Tawhid, "Non-dominated sorting moth flame optimization (NS-MFO) for multi-objective problems," Engineering Applications of Artificial Intelligence, vol. 63, pp. 20-32, 2017.

[33] P. Jangir and N. Jangir, "A new non-dominated sorting grey wolf optimizer (NS-GWO) algorithm: development and application to solve engineering designs and economic constrained emission dispatch problem with integration of wind power," Engineering Applications of Artificial Intelligence, vol. 72, pp. 449-467, 2018.

[34] A. Sadollah, H. Eskandar, and J. H. Kim, "Water cycle algorithm for solving constrained multi-objective optimization problems," Applied Soft Computing, vol. 27, pp. 279-298, 2015.

[35] D.-H. Tran, M.-Y. Cheng, and D. Prayogo, "A novel multiple objective symbiotic organisms Search (MOSOS) for timecost-labor utilization tradeoff problem," Knowledge-Based Systems, vol. 94, pp. 132-145, 2016. 
[36] D. Yu, J. Hong, J. Zhang, and Q. Niu, "Multi-objective individualized-instruction teaching-learning-based optimization algorithm," Applied Soft Computing, vol. 62, pp. 288-314, 2018.

[37] A. Foroughi Nematollahi, A. Rahiminejad, and B. Vahidi, “A novel multi-objective optimization algorithm based on lightning attachment procedure optimization algorithm," Applied Soft Computing, vol. 75, pp. 404-427, 2019.

[38] H. Salimi, "Stochastic fractal search: a powerful metaheuristic algorithm," Knowledge-Based Systems, vol. 75, pp. 1-18, 2015.

[39] R. D. Zimmerman and C. E. Murillo-Sanchez, MatpowerManual-4.1.pdf, Power Systems Engineering Research Center, Cornell University, Ithaca, NY, USA, 2011, https://matpower. org/docs/matpower-manual-4.1.pdf.

[40] K. Buayai, K. Chinnabutr, P. Intarawong, and K. Kerdchuen, "Applied matpower for power system optimization research," Energy Procedia, vol. 56, pp. 505-509, 2014.

[41] W. Sheng, K.-Y. Liu, Y. Liu, X. Meng, and Y. Li, "Optimal placement and sizing of distributed generation via an improved nondominated sorting genetic algorithm II," IEEE Transactions on Power Delivery, vol. 30, no. 2, pp. 569-578, 2015.

[42] D. A. V. Veldhuizen and G. B. Lamont, Multiobjective Evolutionary Algorithm Research: A History and Analysis, Air Force Institute of Technology, Dayton, OH, USA, 1998.

[43] J. R. Schott, Fault Tolerant Design Using Single and Multicriteria Genetic Algorithm Optimization, Massachusetts Institute of Technology, Cambridge, MA, USA, 1995.

[44] K. Deb, Multi-Objective Optimization Using Evolutionary Algorithms, John Wiley \& Sons, Hoboken, NJ, USA, 2001.

[45] E. Zitzler, K. Deb, and L. Thiele, "Comparison of multiobjective evolutionary algorithms: empirical results," Evolutionary Computation, vol. 8, no. 2, pp. 173-195, 2000.

[46] F. Kursawe, "A variant of evolution strategies for vector optimization," in Parallel Problem Solving from Nature, H.-P. Schwefel and R. Männer, Eds., Springer, Berlin, Germany, pp. 193-197, 1991.

[47] M. Laumanns, G. Rudolph, and H.-P. Schwefel, “A spatial predator-prey approach to multi-objective optimization: a preliminary study," in Parallel Problem Solving from Nature-PPSN V, A. E. Eiben, T. Bäck, M. Schoenauer, and H.-P. Schwefel, Eds., Springer, Berlin, Germany, pp. 241-249, 1998.

[48] T. Murata and H. Ishibuchi, "MOGA: multi-objective genetic algorithms," in Proceedings of the 1995 IEEE International Conference on Evolutionary Computation, p. 289, Perth, Australia, 1995.

[49] C. Poloni, A. Giurgevich, L. Onesti, and V. Pediroda, "Hybridization of a multi-objective genetic algorithm, a neural network and a classical optimizer for a complex design problem in fluid dynamics," Computer Methods in Applied Mechanics and Engineering, vol. 186, no. 2, pp. 403-420, 2000.

[50] K. Deb, A. Pratap, S. Agarwal, and T. Meyarivan, "A fast and elitist multiobjective genetic algorithm: NSGA-II," IEEE Transactions on Evolutionary Computation, vol. 6, no. 2, pp. 182-197, 2002.

[51] C. A. C. Coello, G. T. Pulido, and M. S. Lechuga, "Handling multiple objectives with particle swarm optimization," IEEE Transactions on Evolutionary Computation, vol. 8, no. 3, pp. 256-279, 2004.

[52] S. Mirjalili, P. Jangir, S. Z. Mirjalili, S. Saremi, and I. N. Trivedi, "Optimization of problems with multiple objectives using the multi-verse optimization algorithm," Knowledge-Based Systems, vol. 134, pp. 50-71, 2017.
[53] M. E. Baran and F. F. Wu, "Network reconfiguration in distribution systems for loss reduction and load balancing," IEEE Transactions on Power Delivery, vol. 4, no. 2, pp. 14011407, 1989. 